\title{
Formation of Pseudorotaxane Induced by Electrochemical Oxidation of Ferrocene-Containing Axis Molecule in the Presence of Crown Ether
}

\author{
Masaki Horie, Yuji Suzaki, and Kohtaro Osakada* \\ Chemical Resources Laboratory, Tokyo Institute of Technology, \\ 4259 Nagatsuta, Midori-ku, Yokohama 226-8503, Japan
}

\section{Experimental Section}

\section{General Methods}

$\mathbf{1}^{1}$ was prepared according to the literature method. Dibebzo[24]crown-8 (DB24C8) was purchased from Tokyo Kasei Kogyo Co., Ltd. Ferrocenecarboxaldehyde was purchased from STREM CHEMICALS. ${ }^{1} \mathrm{H}$ and ${ }^{13} \mathrm{C}\left\{{ }^{1} \mathrm{H}\right\}$ NMR spectra were recorded on JEOL EX-400 or Varian Mercury 300 spectrometers. The chemical shifts were referenced with respect to $\mathrm{CHCl}_{3}\left(\delta\right.$ 7.24), $\mathrm{CD}_{2} \mathrm{HCN}(\delta 1.93)$ for ${ }^{1} \mathrm{H}$ and $\mathrm{CDCl}_{3}(\delta 77.0)$, $\mathrm{CD}_{3} \mathrm{CN}(\delta 1.30)$ for ${ }^{13} \mathrm{C}$ as internal standards. IR absorption spectra were recorded on Shimadzu FT/IR-8100 spectrometers. Elemental analyses were carried out with a Yanaco MT-5 CHN autorecorder. Thermogravity analysis (TGA) was recorded on Seiko TG/DTA6200R. Differential scanning calorimeter (DSC) was recorded on Seiko DSC6200S. Fast atom bombardment mass spectrum (FABMS) was obtained from JEOL JMS-700 (matrix, glycerol or $m$-nitrobenzyl alcohol). ESR spectra were recorded on a JEOL RE3X spectrometer. Cyclic voltammetry (CV) was measured in $\mathrm{CH}_{3} \mathrm{CN}$ solution containing $10 \mathrm{mM} n-\mathrm{Bu}_{4} \mathrm{NPF}_{6}$ with ALS Electrochemical Analyzer Model-600A. The measurement was carried out in a standard one-compartment cell under inert gas equipped with $\mathrm{Ag}^{+} / \mathrm{Ag}$ reference electrode a platinum-wire counter electrode and a platinum-disk working electrode (ID: $1.6 \mathrm{~mm}$ ). A combination of flow through electrolysis cell, peristaltic pump SMP-11 at $0.7 \mathrm{~mL} / \mathrm{min}$ and JASCO V-530 UV/VIS spectrometer was used for the spectroelectrochemical measurements in situ (Figure S1). ${ }^{2,3}$

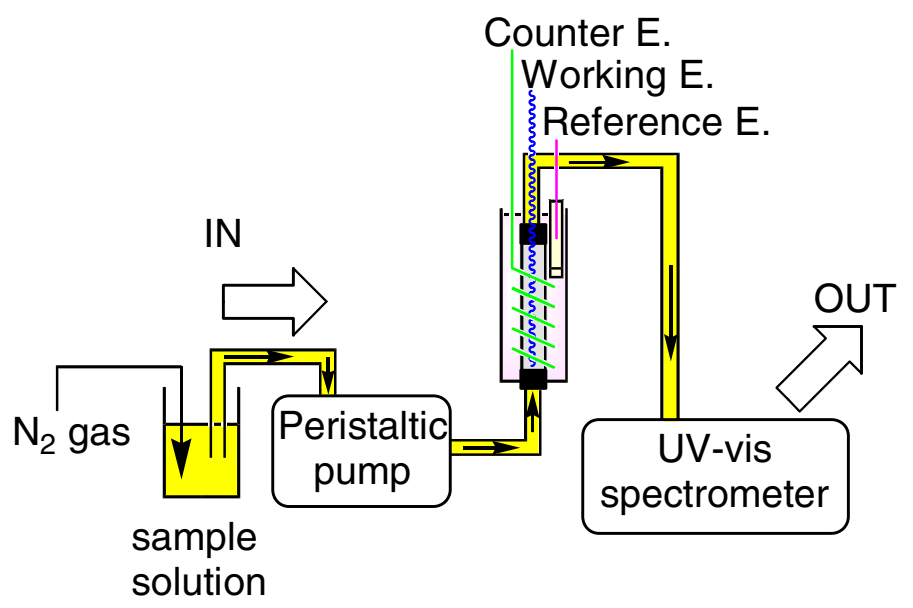

Figure S1. Schematic illustration of flow electrolysis system. 


\section{Preparation of $[1-\mathrm{H}]\left[\mathrm{PF}_{6}\right]$}

A suspension of $1(362 \mathrm{mg}, 1.13 \mathrm{mmol})$ in $6 \mathrm{~N} \mathrm{HCl}(40 \mathrm{~mL})$ was stirred for $12 \mathrm{~h}$ at room temperature. Evaporation of the solvent gave $[1-\mathrm{H}][\mathrm{Cl}]$ which was washed with water. To a suspension of $[1-\mathrm{H}][\mathrm{Cl}]$ in acetone $(50 \mathrm{~mL})$ was added $\mathrm{NH}_{4} \mathrm{PF}_{6}(1.63 \mathrm{~g}, 10 \mathrm{mmol})$, and the mixture was stirred for $4 \mathrm{~h}$ at room temperature. The precipitate was removed by filtration. Evaporation of the filtrate gave $[\mathbf{1}-\mathrm{H}]\left[\mathrm{PF}_{6}\right]$ as a yellow solid $(408 \mathrm{mg}$, $0.877 \mathrm{mmol}, 78 \%$ ). Anal. Calcd. for $\mathrm{C}_{19} \mathrm{H}_{22} \mathrm{NF}_{6} \mathrm{FeP}: \mathrm{C}, 49.06 ; \mathrm{H}, 4.77 ; \mathrm{N}, 3.01$. Found: C, 48.98; H, 4.65; N, 3.02. ${ }^{1} \mathrm{H}$ NMR spectrum ( $300 \mathrm{MHz}, \mathrm{CD}_{3} \mathrm{CN}$, r. t.): $\delta 2.34$ (s, $\left.3 \mathrm{H}, \mathrm{CH}_{3}\right), 4.04\left(\mathrm{br}, 2 \mathrm{H}, \mathrm{CH}_{2}\right), 4.06\left(\mathrm{br}, 2 \mathrm{H}, \mathrm{CH}_{2}\right.$ ), $4.21\left(\mathrm{~s}, 5 \mathrm{H}, \mathrm{C}_{5} \mathrm{H}_{5}\right), 4.28\left(\mathrm{t}, 2 \mathrm{H}, \mathrm{C}_{5} \mathrm{H}_{4}, J(\mathrm{HH})=1.8 \mathrm{~Hz}\right), 4.37\left(\mathrm{t}, 2 \mathrm{H}, \mathrm{C}_{5} \mathrm{H}_{4}, J(\mathrm{HH})=1.8 \mathrm{~Hz}\right), 7.25\left(\mathrm{~d}, 2 \mathrm{H}, \mathrm{C}_{6} \mathrm{H}_{4}, J\right.$ $(\mathrm{HH})=8.1 \mathrm{~Hz}), 7.30\left(\mathrm{~d}, 2 \mathrm{H}, \mathrm{C}_{6} \mathrm{H}_{4}, J(\mathrm{HH})=8.1 \mathrm{~Hz}\right) .{ }^{13} \mathrm{C}\left\{{ }^{1} \mathrm{H}\right\}$ NMR spectrum $\left(75.5 \mathrm{MHz}, \mathrm{CD}_{3} \mathrm{CN}\right.$, r. t. $): \delta 21.3$ $\left(\mathrm{CH}_{3}\right), 48.7\left(\mathrm{CH}_{2}\right), 51.6\left(\mathrm{CH}_{2}\right), 70.1\left(\mathrm{C}_{5} \mathrm{H}_{5}\right.$ or $\left.\mathrm{C}_{5} \mathrm{H}_{4}\right), 70.7\left(\mathrm{C}_{5} \mathrm{H}_{5}\right.$ or $\left.\mathrm{C}_{5} \mathrm{H}_{4}\right), 71.7\left(\mathrm{C}_{5} \mathrm{H}_{5}\right.$ or $\left.\mathrm{C}_{5} \mathrm{H}_{4}\right), 76.5\left(\mathrm{C}_{5} \mathrm{H}_{5}\right.$ or $\left.\mathrm{C}_{5} \mathrm{H}_{4}\right), 128.4\left(\mathrm{C}_{6} \mathrm{H}_{4}\right), 130.6\left(\mathrm{C}_{6} \mathrm{H}_{4}\right), 131.0\left(\mathrm{C}_{6} \mathrm{H}_{4}\right), 140.6\left(\mathrm{C}_{6} \mathrm{H}_{4}\right)$. IR spectrum $(\mathrm{KBr}): v(\mathrm{~N}-\mathrm{H}) ; 3266,3233 \mathrm{~cm}^{-1}$. $5 \%$ weight loss by thermogravimetrys: $217^{\circ} \mathrm{C}$.

\section{Preparation of 2}

A toluene solution $(80 \mathrm{~mL})$ containing ferrocene carboxaldehyde $(2.13 \mathrm{~g}, 10.0 \mathrm{mmol})$ and $n$-hexylamine $(1.02 \mathrm{~g}, 10.1 \mathrm{mmol})$ was stirred for $24 \mathrm{~h}$ at $80^{\circ} \mathrm{C}$ in the presence of MS4A (4 Å molecular sieves). MS4A was removed by filtration and the filtrate was evaporated to give [( $n$-hexylimino)methyl]ferrocene as a brown oil $\left[{ }^{1} \mathrm{H}\right.$ NMR spectrum (300 MHz, $\mathrm{CD}_{3} \mathrm{CN}$, r. t.): $\delta 0.92\left(\mathrm{~m}, 3 \mathrm{H}, \mathrm{CH}_{3}\right), 1.32-1.35 *\left(6 \mathrm{H}, \mathrm{CH}_{2}\right), 1.65\left(\mathrm{~m}, 2 \mathrm{H}, \mathrm{CH}_{2}\right), 3.46$ $\left.\left(2 \mathrm{H}, \mathrm{NCH}_{2}\right), 4.19\left(\mathrm{~s}, 5 \mathrm{H}, \mathrm{C}_{5} \mathrm{H}_{5}\right), 4.36\left(\mathrm{~s}, 2 \mathrm{H}, \mathrm{C}_{5} \mathrm{H}_{4}\right), 4.64\left(\mathrm{~s}, 2 \mathrm{H}, \mathrm{C}_{5} \mathrm{H}_{4}\right), 8.11(\mathrm{~s}, 1 \mathrm{H}, \mathrm{CH})\right]$ The above product was dissolved in THF/MeOH (THF/MeOH $=50 \mathrm{~mL} / 50 \mathrm{~mL}$ ) at room temperature. $\mathrm{NaBH}_{4}(766 \mathrm{mg}, 20.5 \mathrm{mmol})$ was added to the solution and the mixture was stirred for $2 \mathrm{~h}$ at room temperature. An extra portion of $\mathrm{NaBH}_{4}$ (720 mg, $19.0 \mathrm{mmol}$ ) was added to the reaction mixture and the mixture was stirred for further $12 \mathrm{~h}$, before being treated with $1 \mathrm{~N} \mathrm{HCl}(100 \mathrm{~mL})$. After evaporation of the solvent, the remaining solid was partitioned between 2 $\mathrm{N} \mathrm{KOH}$ and $\mathrm{CH}_{2} \mathrm{Cl}_{2}$. Organic extract was dried over $\mathrm{MgSO}_{4}$, filtered, and concentrated under reduced pressure to give [( $n$-hexylamino)methyl]ferrocene as a brown oil $(2.31 \mathrm{~g}, 7.75 \mathrm{mmol}, 78 \%) . \quad{ }^{1} \mathrm{H}$ NMR spectrum (300 $\mathrm{MHz}, \mathrm{CD}_{3} \mathrm{Cl}$, r. t.): $\delta 0.86\left(\mathrm{t}, 3 \mathrm{H}, \mathrm{CH}_{3}, J(\mathrm{HH})=7.2 \mathrm{~Hz}\right), 1.26-1.28^{*}\left(6 \mathrm{H}, \mathrm{CH}_{2}\right), 1.46\left(\mathrm{~m}, 2 \mathrm{H}, \mathrm{CH}_{2}\right), 2.60(\mathrm{t}, 2 \mathrm{H}$, $\left.\mathrm{NCH}_{2}, J(\mathrm{HH})=7.5 \mathrm{~Hz}\right), 3.48\left(\mathrm{~s}, 2 \mathrm{H}, \mathrm{NCH}_{2}\right), 4.08\left(\mathrm{t}, 2 \mathrm{H}, \mathrm{C}_{5} \mathrm{H}_{4}, J(\mathrm{HH})=1.5 \mathrm{~Hz}\right), 4.10\left(\mathrm{~s}, 5 \mathrm{H}, \mathrm{C}_{5} \mathrm{H}_{5}\right), 4.16(\mathrm{t}, 2 \mathrm{H}$, $\left.\mathrm{C}_{5} \mathrm{H}_{4}, J(\mathrm{HH})=1.5 \mathrm{~Hz}\right) .{ }^{13} \mathrm{C}\left\{{ }^{1} \mathrm{H}\right\}$ NMR spectrum $\left(75.5 \mathrm{MHz}, \mathrm{CD}_{3} \mathrm{Cl}\right.$, r. t.): $\delta 14.0\left(\mathrm{CH}_{3}\right), 22.6\left(\mathrm{CH}_{2}\right), 27.1$ $\left(\mathrm{CH}_{2}\right), 30.0\left(\mathrm{CH}_{2}\right), 31.8\left(\mathrm{CH}_{2}\right), 49.1\left(\mathrm{NCH}_{2}\right), 49.7\left(\mathrm{NCH}_{2}\right), 67.7\left(\mathrm{C}_{5} \mathrm{H}_{4}\right.$ or $\left.\mathrm{C}_{5} \mathrm{H}_{5}\right), 68.2-68.4 *\left(2 \mathrm{C}, \mathrm{C}_{5} \mathrm{H}_{4}\right.$ or $\left.\mathrm{C}_{5} \mathrm{H}_{5}\right)$, $68.4\left(\mathrm{C}_{5} \mathrm{H}_{4}\right.$ or $\left.\mathrm{C}_{5} \mathrm{H}_{5}\right)$. The peaks with asterisks are overlapped significantly with other signals.

\section{Preparation of $[2-\mathrm{H}]\left[\mathrm{PF}_{6}\right]$}

A suspension of $2(740 \mathrm{mg}, 2.47 \mathrm{mmol})$ in $6 \mathrm{~N} \mathrm{HCl}(60 \mathrm{~mL})$ was stirred for $12 \mathrm{~h}$ at room temperature. Evaporation of the solvent gave $[2-\mathrm{H}][\mathrm{Cl}]$ which was washed with water. To a suspension of $[2-\mathrm{H}][\mathrm{Cl}]$ in acetone $(50 \mathrm{~mL})$ was added $\mathrm{NH}_{4} \mathrm{PF}_{6}(1.63 \mathrm{~g}, 10 \mathrm{mmol})$ and the mixture was stirred for $4 \mathrm{~h}$ at room temperature. The precipitate was removed by filtration and the evaporation of the filtrate gave $[\mathbf{2}-\mathrm{H}]\left[\mathrm{PF}_{6}\right]$ as a yellow solid (395 mg, 0.887 mmol, 36\%). Anal. Calcd. for $\mathrm{C}_{17} \mathrm{H}_{26} \mathrm{NF}_{6} \mathrm{FeP}: \mathrm{C}, 45.86 ; \mathrm{H}, 5.89 ; \mathrm{N}, 3.15$. Found: $\mathrm{C}, 45.98 ; \mathrm{H}$, 6.03; N, 3.20. ${ }^{1} \mathrm{H}$ NMR spectrum (300 MHz, $\mathrm{CD}_{3} \mathrm{CN}$, r. t.): $\delta 0.88\left(\mathrm{t}, 3 \mathrm{H}, \mathrm{CH}_{3}, J(\mathrm{HH})=6.9 \mathrm{~Hz}\right), 1.28-1.32 *$ $\left(6 \mathrm{H}, \mathrm{CH}_{2}\right), 1.57\left(\mathrm{~m}, 2 \mathrm{H}, \mathrm{CH}_{2}\right), 2.90\left(\mathrm{~m}, 2 \mathrm{H}, \mathrm{NCH}_{2} \mathrm{CH}_{2}\right), 3.97\left(\mathrm{~s}, 2 \mathrm{H}, \mathrm{NCH}_{2} \mathrm{C}_{5} \mathrm{H}_{4}\right), 4.20\left(\mathrm{~s}, 5 \mathrm{H}, \mathrm{C}_{5} \mathrm{H}_{5}\right), 4.27(\mathrm{t}, 2 \mathrm{H}$, $\left.\mathrm{C}_{5} \mathrm{H}_{4}, J(\mathrm{HH})=2.1 \mathrm{~Hz}\right), 4.35\left(\mathrm{t}, 2 \mathrm{H}, \mathrm{C}_{5} \mathrm{H}_{4}, J(\mathrm{HH})=1.8 \mathrm{~Hz}\right), 6.41\left(\mathrm{br}, 2 \mathrm{H}, \mathrm{NH}_{2}\right) .{ }^{13} \mathrm{C}\left\{{ }^{1} \mathrm{H}\right\} \mathrm{NMR}$ spectrum $(75.5$ $\mathrm{MHz}, \mathrm{CD}_{3} \mathrm{CN}$, r. t.): $\delta 14.2\left(\mathrm{CH}_{3}\right), 23.0\left(\mathrm{CH}_{2}\right), 26.3\left(\mathrm{CH}_{2}\right), 26.5\left(\mathrm{CH}_{2}\right), 31.7\left(\mathrm{CH}_{2}\right), 48.2\left(\mathrm{NCH}_{2}\right), 48.7\left(\mathrm{NCH}_{2}\right)$, $69.9\left(\mathrm{C}_{5} \mathrm{H}_{5}\right.$ or $\left.\mathrm{C}_{5} \mathrm{H}_{4}\right), 70.5\left(\mathrm{C}_{5} \mathrm{H}_{5}\right.$ or $\left.\mathrm{C}_{5} \mathrm{H}_{4}\right), 71.3\left(\mathrm{C}_{5} \mathrm{H}_{5}\right.$ or $\left.\mathrm{C}_{5} \mathrm{H}_{4}\right), 76.6\left(\mathrm{C}_{5} \mathrm{H}_{5}\right.$ or $\left.\mathrm{C}_{5} \mathrm{H}_{4}\right)$. The peak with asterisk is overlapped significantly with other signals. 


\section{Isolation of [DB24C8・1-H] $\left[\mathrm{PF}_{6}\right]$}

Yellow crystals of pseudorotaxane $[\mathrm{DB} 24 \mathrm{C} 8 \cdot 1-\mathrm{H}]\left[\mathrm{PF}_{6}\right]$ was obtained by recrystallization from a $\mathrm{CH}_{2} \mathrm{Cl}_{2} / \mathrm{Et}_{2} \mathrm{O}$ solution of $[1-\mathrm{H}]\left[\mathrm{PF}_{6}\right] / \mathrm{DB} 24 \mathrm{C} 8\left([1-\mathrm{H}]\left[\mathrm{PF}_{6}\right] / \mathrm{DB} 24 \mathrm{C} 8=58.3 \mathrm{mg} / 46.5 \mathrm{mg}\right) . \quad[\mathrm{DB} 24 \mathrm{C} 8 \cdot 1-\mathrm{H}]\left[\mathrm{PF}_{6}\right]$ was obtained in 72\%. Anal. Calcd. for $\mathrm{C}_{43} \mathrm{H}_{54} \mathrm{NF}_{6} \mathrm{FeP}: \mathrm{C}, 56.52 ; \mathrm{H}, 5.96 ; \mathrm{N}, 1.53$. Found: $\mathrm{C}, 56.37 ; \mathrm{H}, 5.80 ; \mathrm{N}$, 1.53. IR spectrum (KBr): $v(\mathrm{~N}-\mathrm{H}): 3067,3166 \mathrm{~cm}^{-1}$. FABMS: $\mathrm{m} / z=768\left[\mathrm{M}-\mathrm{PF}_{6}\right]^{+}$. $5 \%$ weight loss by thermogravimetrys: temperature $237^{\circ} \mathrm{C}$.

\section{Crystal structure analysis}

Crystals of $[\mathrm{DB} 24 \mathrm{C} 8 \bullet 1-\mathrm{H}]\left[\mathrm{PF}_{6}\right]$ suitable for X-ray diffraction study were obtained by recrystallization from $\mathrm{CH}_{2} \mathrm{Cl}_{2} / \mathrm{Et}_{2} \mathrm{O}$ and mounted in glass capillary tubes. The data was collected to a maximum $2 \theta$ value of 55.0 ․ A total of 720 oscillation images were collected. A sweep of data was done using $\omega$ scans from -110.0 to $70.0^{\circ}$ in $0.5^{\circ}$ step, at $\chi=45.0^{\circ}$ and $\phi=0.0^{\circ}$. The detector swing angle was $-20.42^{\circ}$. A second sweep was performed using $\omega$ scans from -110.0 to $70.0^{\circ}$ in $0.5^{\circ}$ step, at $\chi=45.0^{\circ}$ and $\phi=90.0^{\circ}$. The crystal-to-detector distance was $44.84 \mathrm{~mm}$. Readout was performed in the $0.070 \mathrm{~mm}$ pixel mode. Calculations were carried out by using a program package CrystalStructure ${ }^{\mathrm{TM}}$ for Windows. The structure was solved by direct methods and expanded using Fourier techniques. A full-matrix least-squares refinement was used for the non-hydrogen atoms with anisotropic thermal parameters.

\section{Pseudo[2]rotaxane formation in $\mathrm{CD}_{3} \mathrm{CN}$}

In an NMR tube was charged a $\mathrm{CD}_{3} \mathrm{CN}$ solution $(0.7 \mathrm{~mL})$ of DB24C8 $(0.007 \mathrm{mmol})$ and the ammonium salts $\left([\mathbf{1}-\mathrm{H}]\left[\mathrm{PF}_{6}\right]\right.$ or $\left.[\mathbf{2}-\mathrm{H}]\left[\mathrm{PF}_{6}\right], 0.007 \mathrm{mmol}\right)$. The sample was placed in an NMR spectrometer. The ${ }^{1} \mathrm{H}$ NMR spectra were recorded at $30^{\circ} \mathrm{C}$ (Figure S2).

The molar ratios of $[\mathrm{DB} 24 \mathrm{C} 8 \cdot \mathbf{1}-\mathrm{H}]\left[\mathrm{PF}_{6}\right]$ to $[\mathbf{1}-\mathrm{H}]\left[\mathrm{PF}_{6}\right]$ or $[\mathrm{DB} 24 \mathrm{C} 8 \cdot \mathbf{2}-\mathrm{H}]\left[\mathrm{PF}_{6}\right]$ to $[\mathbf{2}-\mathrm{H}]\left[\mathrm{PF}_{6}\right]$ are determined by the comparison of the peak area of methyl hydrogens or methylene hydrogens: $[\mathrm{DB} 24 \mathrm{C} 8 \bullet \mathbf{1}-\mathrm{H}]\left[\mathrm{PF}_{6}\right] /[\mathbf{1}-\mathrm{H}]\left[\mathrm{PF}_{6}\right]=2.0 / 1.6,[\mathrm{DB} 24 \mathrm{C} 8 \bullet \mathbf{2}-\mathrm{H}]\left[\mathrm{PF}_{6}\right] /[\mathbf{2}-\mathrm{H}]\left[\mathrm{PF}_{6}\right]=1.0 / 1.0$.

${ }^{1} \mathrm{H}$ NMR spectrum of [DB24C8・1-H] $\left[\mathrm{PF}_{6}\right]\left(300 \mathrm{MHz}, \mathrm{CD}_{3} \mathrm{CN}\right.$, r. t.): $\delta 2.14$ (s, 3H, $\left.\mathrm{CH}_{3}\right), 3.60-4.20 *$ $\left(\mathrm{OCH}_{2}, \mathrm{C}_{5} \mathrm{H}_{5}, \mathrm{C}_{5} \mathrm{H}_{4}, 33 \mathrm{H}\right), 4.37\left(\mathrm{~m}, 2 \mathrm{H}, \mathrm{NCH}_{2}\right), 4.53\left(\mathrm{~m}, 2 \mathrm{H}, \mathrm{NCH}_{2}\right), 6.85-6.64 *\left(10 \mathrm{H}, \mathrm{C}_{6} \mathrm{H}_{4}, o-\right.$ or $m-\mathrm{C}_{6} \mathrm{H}_{4}$ (host)), $7.14\left(2 \mathrm{H}, o\right.$ - or $m-\mathrm{C}_{6} \mathrm{H}_{4}$ (host)). ${ }^{1} \mathrm{H}$ NMR spectrum of [DB24C8 $\left.\bullet 2-\mathrm{H}\right]\left[\mathrm{PF}_{6}\right]\left(400 \mathrm{MHz}, \mathrm{CD}_{3} \mathrm{CN}\right.$, $\left.30{ }^{\circ} \mathrm{C}\right): \delta 0.89\left(\mathrm{t}, 3 \mathrm{H}, \mathrm{CH}_{3}, J(\mathrm{HH})=0.8 \mathrm{~Hz}\right), 1.03-1.11 *\left(4 \mathrm{H}, \mathrm{CH}_{2}\right), 1.29-1.38 *\left(2 \mathrm{H}, \mathrm{CH}_{2}\right), 1.40\left(\mathrm{~m}, 2 \mathrm{H}, \mathrm{CH}_{2}\right)$, $3.32\left(\mathrm{~m}, 2 \mathrm{H}, \mathrm{NCH}_{2}\right), 3.60-4.35 *\left(35 \mathrm{H}, \mathrm{C}_{5} \mathrm{H}_{5}, \mathrm{OCH}_{2}\right), 6.88-6.98 *\left(8 \mathrm{H}, \mathrm{C}_{6} \mathrm{H}_{4}\right)$. FABMS: $m / z=748\left[\mathrm{M}-\mathrm{PF}_{6}\right]^{+}$. The peaks with asterisks are overlapped significantly with other signals.

\section{Kinetic study of pseudo[2]rotaxane formation}

Typical kinetic experiment is as follows. An acetonitrile solution of $\mathbf{1}(2 \mathrm{mM})$ containing $n-\mathrm{Bu}_{4} \mathrm{NPF}_{6}$ $(0.1 \mathrm{M})$ was electrochemically oxidized at $0.4 \mathrm{~V}\left(\mathrm{vs} \mathrm{Ag}^{+} / \mathrm{Ag}\right)$ by using flow electrolysis system and was soon collect to $1 \mathrm{~cm} \times 1 \mathrm{~cm}$ quartz cell. After addition of DB24C8 $(0,4,10,20$, or $40 \mathrm{mM})$ to the solution, the $\mathrm{UV}$-vis absorption spectra were measured every 3 minutes. 


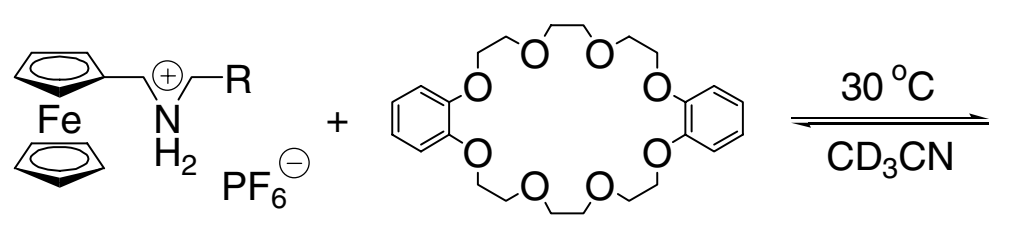

$\left[[1-\mathrm{H}]\left[\mathrm{PF}_{6}\right]\right]_{0},\left[[2-\mathrm{H}]\left[\mathrm{PF}_{6}\right]\right]_{0}=[\mathrm{DB} 24 \mathrm{C} 8]_{0}=10 \mathrm{mM}$

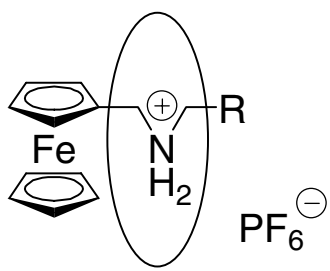

$[\mathrm{DB} 24 \mathrm{C} 8 \cdot 1-\mathrm{H}]\left[\mathrm{PF}_{6}\right]$ $[\mathrm{DB} 24 \mathrm{C} 8 \cdot 2-\mathrm{H}]\left[\mathrm{PF}_{6}\right]$

$1-\mathrm{H} \quad \mathrm{R}=-\xi-\mathrm{Me}$

$2-\mathrm{HR}=-\mathrm{\xi}^{-}$

(a)

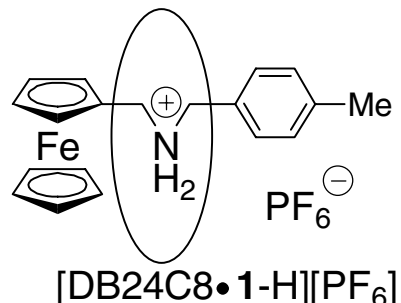

$[\mathrm{DB} 24 \mathrm{C} 8 \bullet 1-\mathrm{H}]\left[\mathrm{PF}_{6}\right]$

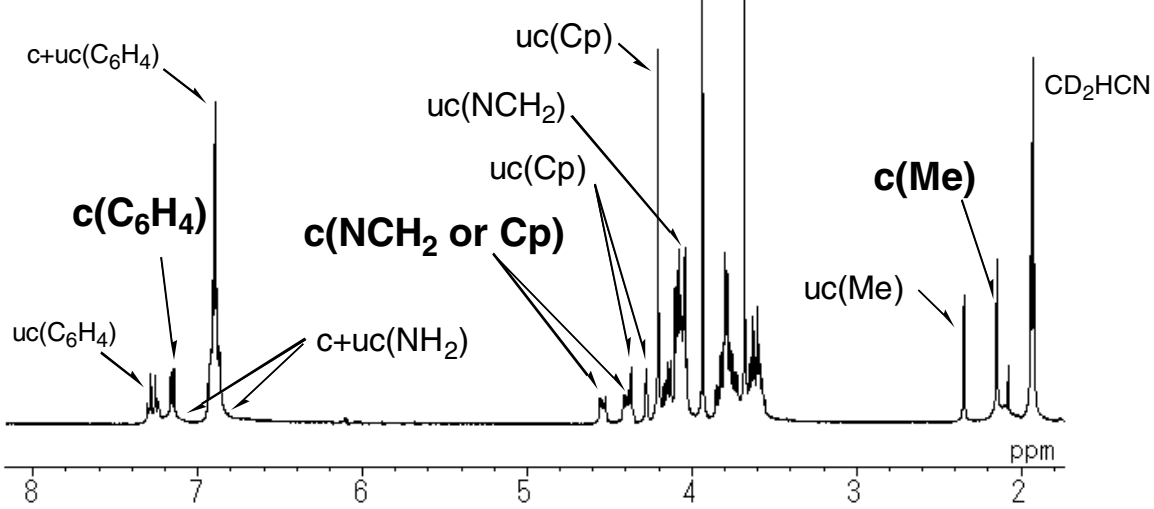

(b)

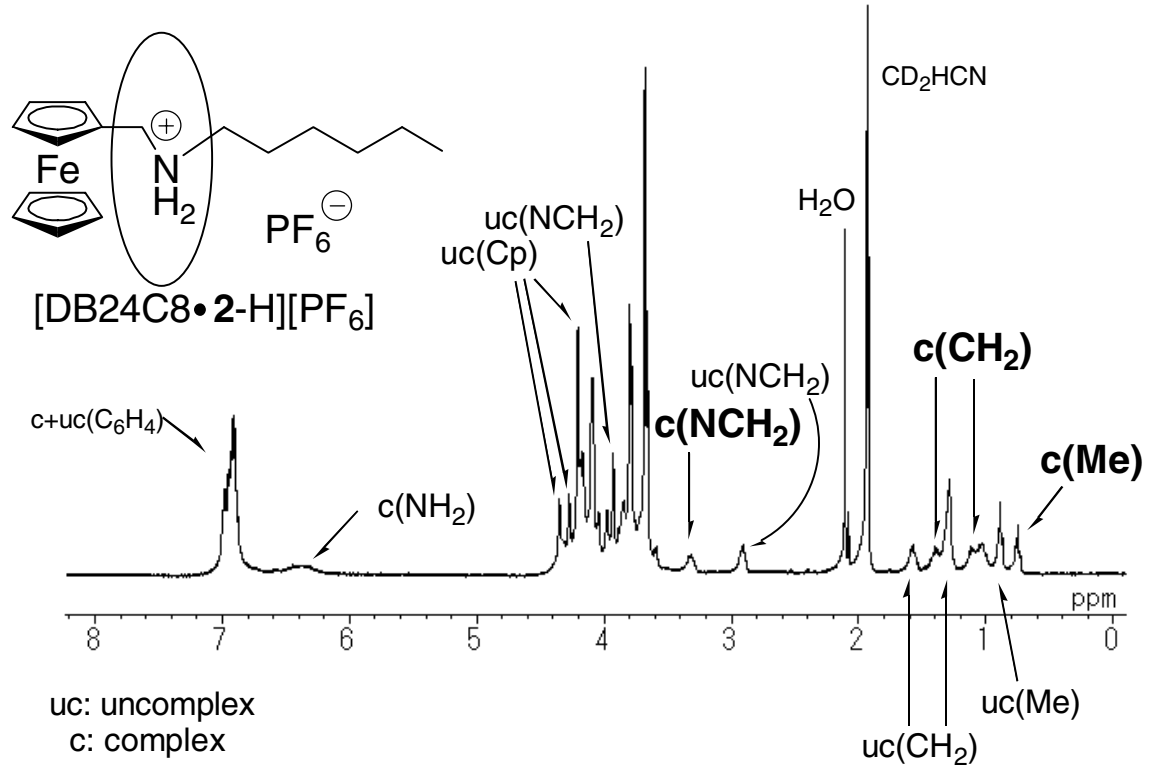

Figure S2. ${ }^{1} \mathrm{H} \mathrm{NMR}$ spectra in $\mathrm{CD}_{3} \mathrm{CN}\left(400 \mathrm{MHz}, 30{ }^{\circ} \mathrm{C}\right)$. 


\section{${ }^{1} \mathrm{H}$ NMR spectra of mixture of $1, n-\mathrm{Bu}_{4} \mathrm{NPF}_{6}, \mathrm{TEMPOH}$, and DB24C8}

${ }^{1} \mathrm{H}$ NMR spectrum (300 MHz, r. t.) of $50 \mathrm{mM}$ of $\mathbf{1}$ was measured in $\mathrm{CD}_{3} \mathrm{CN}$ (Figure S3). After addition of $50 \mathrm{mM}$ of $n-\mathrm{Bu}_{4} \mathrm{NPF}_{6}, \mathrm{TEMPOH}$, and DB24C8 these spectra showed that any reaction without electrochemical oxidation.

(a)
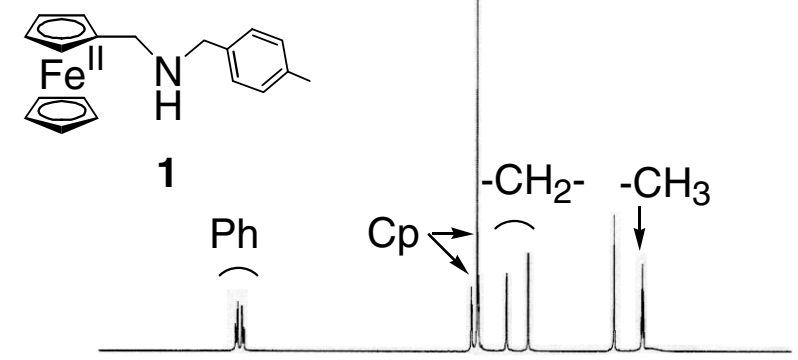

(b) $+n-\mathrm{Bu}_{4} \mathrm{NPF}_{6}$

(c)

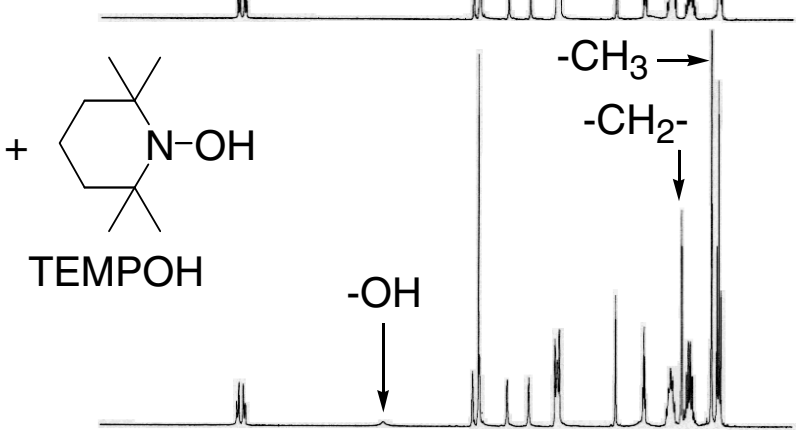

(d)

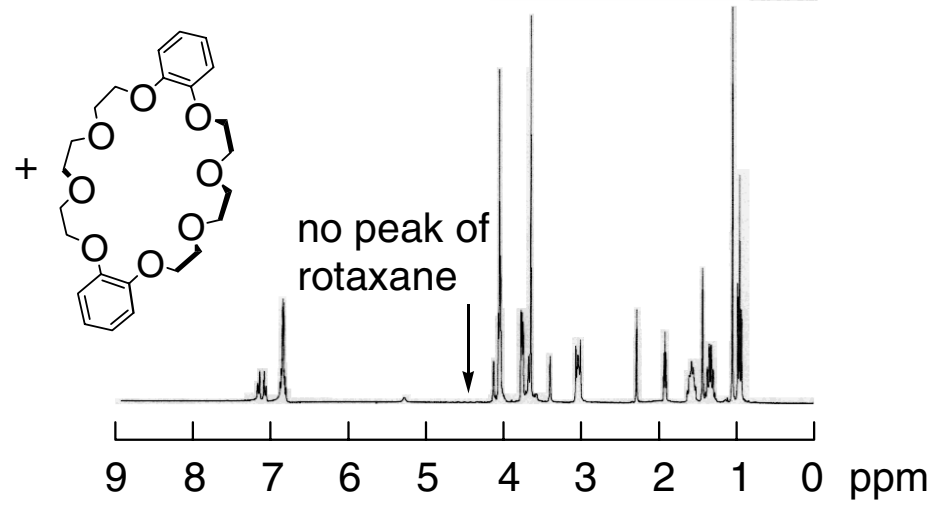

Figure S3. ${ }^{1} \mathrm{H}$ NMR spectra of (a) $\mathbf{1}$, by addition of (b) $n-\mathrm{Bu}_{4} \mathrm{NPF}_{6}$, (c) $n-\mathrm{Bu}_{4} \mathrm{NPF}_{6}$ and TEMPOH and (d) $n$-Bu $\mathrm{NPF}_{6}$, TEMPOH and DB24C8 in $\mathrm{CD}_{3} \mathrm{CN}(300 \mathrm{MHz}$, r. t.). 
(a)
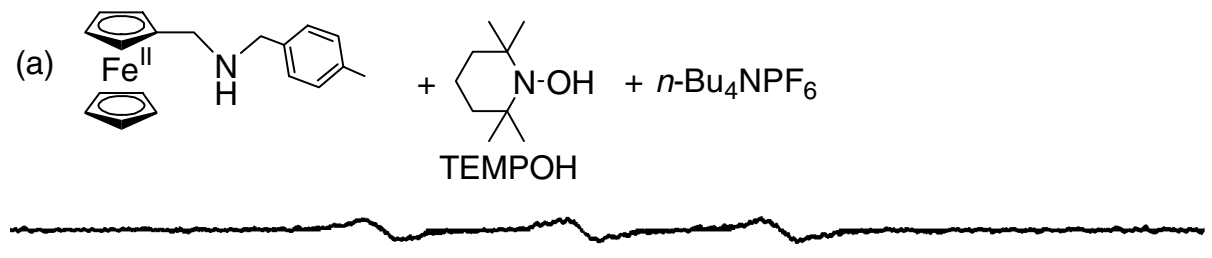

(b)
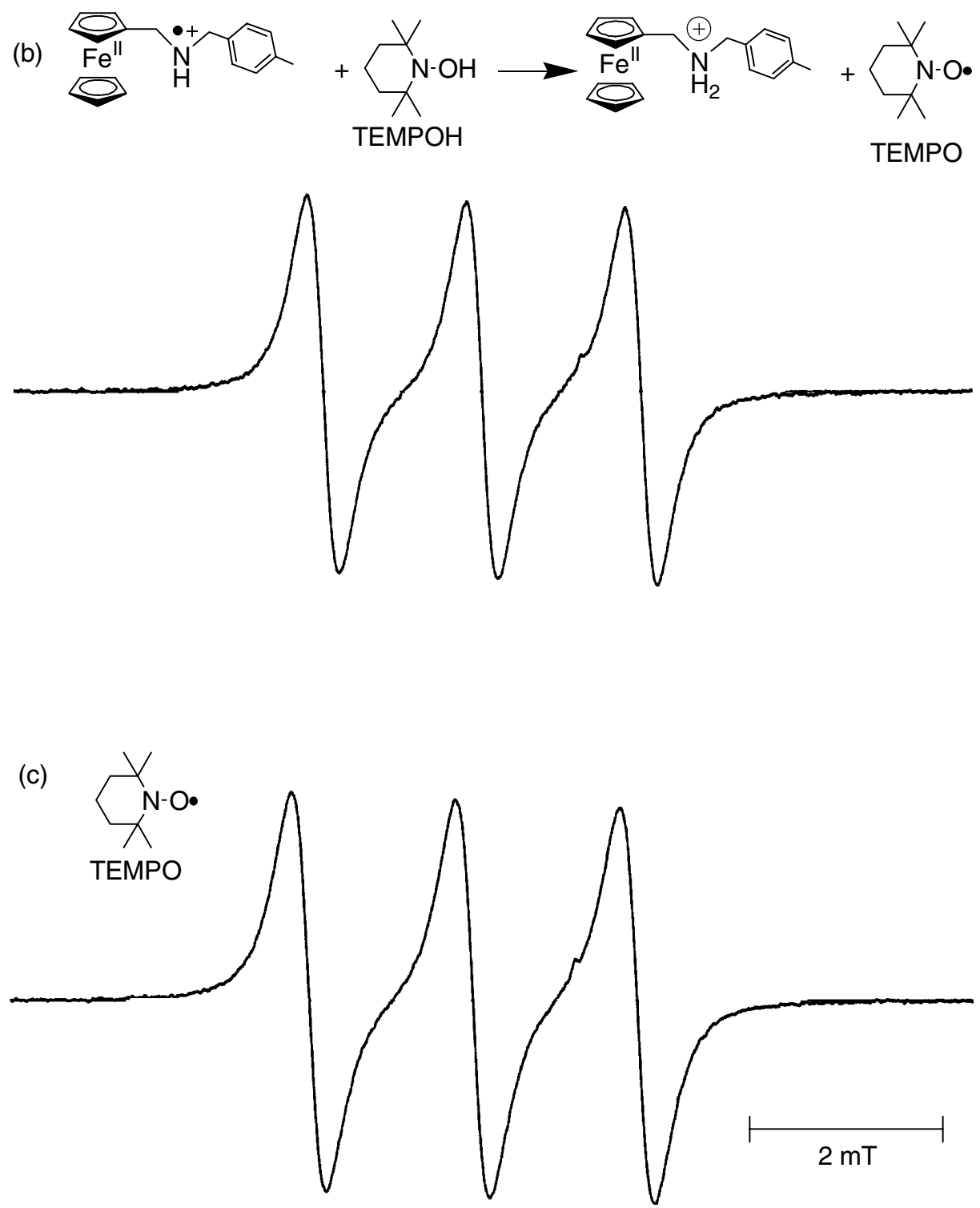

Figure S4. ESR spectra of (a) mixture of $1(1 \mathrm{mM}), n-\mathrm{Bu}_{4} \mathrm{NPF}_{6}(1 \mathrm{mM})$ and TEMPOH $(1 \mathrm{mM})$, (b) mixture of $1(1 \mathrm{mM})$ and $n-\mathrm{Bu}_{4} \mathrm{NPF}_{6}(1 \mathrm{mM})$ after flow electrolysis at $0.4 \mathrm{~V}$ and addition of TEMPOH $(1 \mathrm{mM})$ and (c) TEMPO radical $(1 \mathrm{mM})$. Integral intensity ratio $(\mathrm{b} / \mathrm{c})=0.92$. 


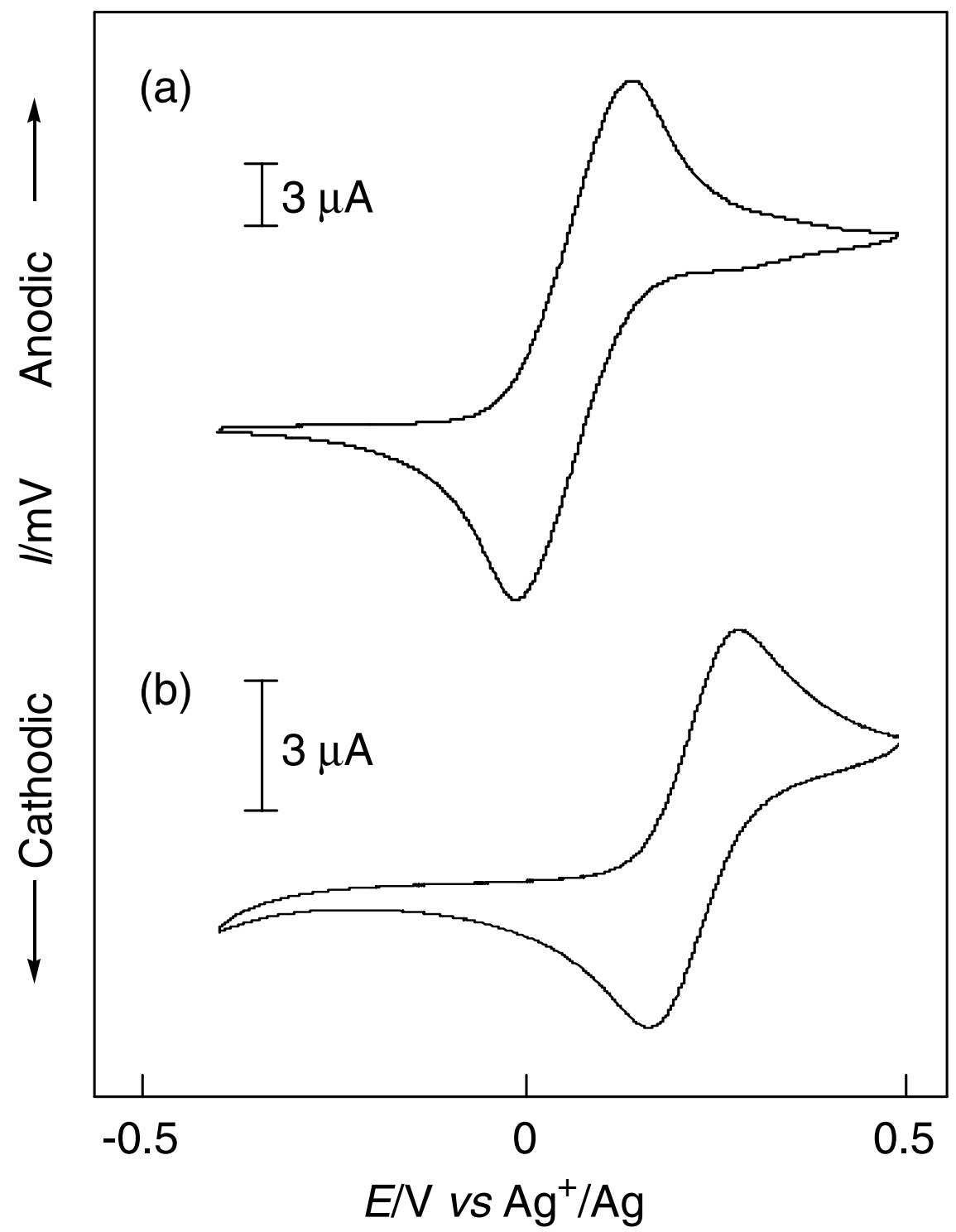

Figure S5. Cyclic voltammograms of $1(2 \mathrm{mM})$ in the presence of DB24C8 $(4 \mathrm{mM})$ in acetonitrile solution containing $10 \mathrm{mM} n-\mathrm{Bu}_{4} \mathrm{NPF}_{6}$ at scanning rates of $0.1 \mathrm{Vs}^{-1}$, (a) before flow electrolysis, (b) after flow electrolysis at $0.4 \mathrm{~V}$ and kept at $20^{\circ} \mathrm{C}$ for $12 \mathrm{~h}$.

\section{References}

1. Hess, A., Brosch, O., Weyhermuller, T.; Metzler-Nolte, N. J. Organomet. Chem. 1999, 589, 75.

2. Nakao, H. Hayashi, H.; Okita, K. Anal. Sci. 2001, 17, 545.

3. Sakano, T., Horie, M., Osakada, K.; Nakao, H. Bull. Chem. Soc. Jpn. 2001, 74, 2059. 


\section{X-ray Structure Report for $[\mathrm{DB} 24 \mathrm{C} 8 \cdot 1-\mathrm{H}]\left[\mathrm{PF}_{6}\right]$}

\section{Experimental}

\section{Data Collection}

A yellow prism crystal of $\mathrm{C}_{43} \mathrm{H}_{54} \mathrm{~F}_{6} \mathrm{FeNO}_{8} \mathrm{P}$ having approximate dimensions of $0.50 \times 0.20 \times 0.15 \mathrm{~mm}$ was mounted on a glass fiber. All measurements were made on a Rigaku Saturn CCD area detector with graphite monochromated Mo-K $\alpha$ radiation.

Indexing was performed from 0 images that were exposed for 0 seconds. The crystal-to-detector distance was $44.84 \mathrm{~mm}$. dimensions:

Cell constants and an orientation matrix for data collection corresponded to a primitive triclinic cell with

$$
\begin{array}{lll}
\mathrm{a}= & 10.1889(13) \AA & \alpha=87.09(3)^{\mathrm{O}} \\
\mathrm{b}= & 11.050(3) \AA & \beta=77.37(2)^{\mathrm{O}} \\
\mathrm{c}= & 19.460(2) \AA & \gamma=89.02(2)^{\mathrm{O}} \\
\mathrm{V}=2135.1(7) \AA^{3} &
\end{array}
$$

For $\mathrm{Z}=2$ and F.W. $=913.71$, the calculated density is $1.42 \mathrm{~g} / \mathrm{cm}^{3}$. Based on a statistical analysis of intensity distribution, and the successful solution and refinement of the structure, the space group was determined to be:

$$
\text { P-1 (\#2) }
$$

The data were collected at a temperature of $-180+1^{\circ} \mathrm{C}$ to a maximum $2 \theta$ value of $55.0^{\circ}$. A total of 720 oscillation images were collected. A sweep of data was done using $\omega$ scans from -110.0 to $70.0^{\circ}$ in $0.5^{\circ}$ step, at $\chi=45.0^{\circ}$ and $\phi=0.0^{\circ}$. The exposure rate was $30.0\left[\mathrm{sec} . /^{\mathrm{O}}\right]$. The detector swing angle was $-20.42^{\mathrm{O}}$. A second sweep was performed using $\omega$ scans from -110.0 to $70.0^{\circ}$ in $0.5^{\circ}$ step, at $\chi=45.0^{\circ}$ and $\phi=90.0^{\circ}$. The exposure rate was 30.0 $\left[\mathrm{sec} . /^{\mathrm{O}}\right]$. The detector swing angle was $-20.42^{\mathrm{O}}$. The crystal-to-detector distance was $44.84 \mathrm{~mm}$. Readout was performed in the $0.070 \mathrm{~mm}$ pixel mode.

\section{Data Reduction}

Of the 14967 reflections that were collected, 8838 were unique $\left(\mathrm{R}_{\mathrm{int}}=0.061\right)$; equivalent reflections were merged. Data were collected and processed using CrystalClear (Rigaku). Net intensities and sigmas were derived as follows:

$$
\begin{aligned}
& \mathrm{F}^{2}=\left[\Sigma\left(\mathrm{P}_{\mathrm{i}}-\mathrm{mB}_{\mathrm{ave}}\right)\right] \cdot \mathrm{Lp}^{-1} \\
& \text { where } P_{i} \text { is the value in counts of the } i^{\text {th }} \text { pixel } \\
& \mathrm{m} \text { is the number of pixels in the integration area } \\
& B_{\text {ave }} \text { is the background average } \\
& \mathrm{Lp} \text { is the Lorentz and polarization factor } \\
& \mathrm{B}_{\text {ave }}=\Sigma\left(\mathrm{B}_{\mathrm{j}}\right) / \mathrm{n} \\
& \text { where } \mathrm{n} \text { is the number of pixels in the background area } \\
& \mathrm{B}_{\mathrm{j}} \text { is the value of the } \mathrm{j}^{\text {th }} \text { pixel in counts } \\
& \Sigma^{2}\left(\mathrm{~F}^{2} \mathrm{hkl}\right)=\left[\left(\Sigma \mathrm{P}_{\mathrm{i}}\right)+\mathrm{m}\left(\left(\Sigma\left(\mathrm{B}_{\mathrm{ave}}-\mathrm{B}_{\mathrm{j}}\right)^{2}\right) /(\mathrm{n}-1)\right)\right] \cdot \quad \mathrm{Lp} \cdot \quad \text { errmul }+\left(\text { erradd } \cdot \mathrm{F}^{2}\right)^{2} \\
& \text { where erradd }=0.00 \\
& \text { errmul }=1.00
\end{aligned}
$$

The linear absorption coefficient, $\mu$, for Mo-K $\alpha$ radiation is $4.7 \mathrm{~cm}^{-1}$. was applied which resulted in transmission factors ranging from 0.77 to 1.00 . The data were corrected for Lorentz and polarization effects.

\section{$\underline{\text { Structure Solution and Refinement }}$}

The structure was solved by direct methods ${ }^{2}$ and expanded using Fourier techniques ${ }^{3}$. The non-hydrogen 
atoms were refined anisotropically. Hydrogen atoms were refined using the riding model. The final cycle of full-matrix least-squares refinement ${ }^{4}$ on $\mathrm{F}$ was based on 5174 observed reflections $(\mathrm{I}>3.00 \Sigma(\mathrm{I})$ ) and 595 variable parameters and converged (largest parameter shift was 0.01 times its esd) with unweighted and weighted agreement factors of:

$$
\begin{gathered}
\mathrm{R}=\Sigma\|\mathrm{Fo}|-| \mathrm{Fc}\| / \Sigma|\mathrm{Fo}|=0.063 \\
\mathrm{R}_{\mathrm{W}}=\left[\Sigma \mathrm{w}(|\mathrm{Fo}|-|\mathrm{Fc}|)^{2} / \Sigma \mathrm{w} \mathrm{Fo}^{2}\right]^{1 / 2}=0.090
\end{gathered}
$$

The standard deviation of an observation of unit weight ${ }^{5}$ was 0.95 . A Sheldrick weighting scheme was used. Plots of $\Sigma \mathrm{w}(|\mathrm{Fo}|-|\mathrm{Fc}|)^{2}$ versus $|\mathrm{Fo}|$, reflection order in data collection, sin $\theta / \lambda$ and various classes of indices showed no unusual trends. The maximum and minimum peaks on the final difference Fourier map corresponded to 1.48 and $-0.66 \mathrm{e}^{-} / \AA^{3}$, respectively.

Neutral atom scattering factors were taken from Cromer and Waber ${ }^{6}$. Anomalous dispersion effects were included in $\mathrm{Fcalc}^{7}$; the values for $\Delta \mathrm{f}^{\prime}$ and $\Delta \mathrm{f}^{\prime \prime}$ were those of Creagh and McAuley ${ }^{8}$. The values for the mass attenuation coefficients are those of Creagh and Hubbell ${ }^{9}$. All calculations were performed using the CrystalStructure ${ }^{10,11}$ crystallographic software package.

\section{References}

(1) CrystalClear: Rigaku Corporation, 1999. CrystalClear Software User's Guide, Molecular Structure Corporation, (c) 2000.J.W.Pflugrath (1999) Acta Cryst. D55, 1718-1725.

(2) SIR92: Altomare, A., Cascarano, G., Giacovazzo, C., Guagliardi, A., Burla, M., Polidori, G., and Camalli, M. (1994) J. Appl. Cryst., 27, 435.

(3) DIRDIF99: Beurskens, P.T., Admiraal, G., Beurskens, G., Bosman, W.P., de Gelder, R., Israel, R. and Smits, J.M.M.(1999). The DIRDIF-99 program system, Technical Report of the Crystallography Laboratory, University of Nijmegen, The Netherlands.

(4) Least Squares function minimized:

$$
\Sigma w\left(\left|\mathrm{~F}_{\mathrm{O}}\right|-\left|\mathrm{F}_{\mathrm{C}}\right|\right)^{2} \quad \text { where } \mathrm{w}=\text { Least Squares weights. }
$$

(5) Standard deviation of an observation of unit weight:

$$
\begin{aligned}
& {\left[\Sigma w\left(\left|\mathrm{~F}_{\mathrm{O}}\right|-\left|\mathrm{F}_{\mathrm{C}}\right|\right)^{2} /\left(\mathrm{N}_{\mathrm{O}}-\mathrm{N}_{\mathrm{V}}\right)\right]^{1 / 2}} \\
& \text { where: } \quad \mathrm{N}_{\mathrm{O}}=\text { number of observations } \\
& \qquad \mathrm{N}_{\mathrm{V}}=\text { number of variables }
\end{aligned}
$$

(6) Cromer, D. T. \& Waber, J. T.; "International Tables for X-ray Crystallography", Vol. IV, The Kynoch Press, Birmingham, England, Table 2.2 A (1974).

(7) Ibers, J. A. \& Hamilton, W. C.; Acta Crystallogr., 17, 781 (1964).

(8) Creagh, D. C. \& McAuley, W.J .; "International Tables for Crystallography", Vol C, (A.J.C. Wilson, ed.), Kluwer Academic Publishers, Boston, Table 4.2.6.8, pages 219-222 (1992).

(9) Creagh, D. C. \& Hubbell, J.H..; "International Tables for Crystallography", Vol C, (A.J.C. Wilson, ed.), Kluwer Academic Publishers, Boston, Table 4.2.4.3, pages 200-206 (1992).

(10) CrystalStructure 3.10: Crystal Structure Analysis Package, Rigaku and Rigaku/MSC (2000-2002).

(11) CRYSTALS Issue 10: Watkin, D.J., Prout, C.K. Carruthers, J.R. \& Betteridge, P.W. Chemical Crystallography Laboratory, Oxford, UK. 
A. Crystal Data

Empirical Formula

Formula Weight

Crystal Color, Habit

Crystal Dimensions

Crystal System

Lattice Type

Detector Position

Pixel Size

Lattice Parameters

Space Group

$Z$ value

$\mathrm{D}_{\text {calc }}$

F000

$\mu(\operatorname{MoK} \alpha)$

Detector

Goniometer

Radiation

Detector Aperture

Data Images

$\omega$ oscillation Range $(\chi=45.0, \phi=0.0)$

Exposure Rate

Detector Swing Angle

$\omega$ oscillation Range $(\chi=45.0, \phi=90.0)$

Exposure Rate

Detector Swing Angle

Detector Position

Pixel Size

$2 \theta_{\max }$

No. of Reflections Measured

Corrections

$\mathrm{C}_{43} \mathrm{H}_{54} \mathrm{~F}_{6} \mathrm{FeNO}_{8} \mathrm{P}$
913.71
yellow, prism
$0.50 \mathrm{X} 0.20 \mathrm{X} 0.15 \mathrm{~mm}$
triclinic
Primitive
$44.84 \mathrm{~mm}$
$0.035 \mathrm{~mm}$
$\mathrm{a}=10.1889(13) \AA$
$\mathrm{b}=11.050(3) \AA$
$\mathrm{c}=19.460(2) \AA$
$\alpha=87.09(3)^{\mathrm{O}}$
$\beta=77.37(2)^{\mathrm{O}}$
$\gamma=89.02(2)^{\mathrm{o}}$
$\mathrm{V}=2135.1(7) \AA^{3}$
$\mathrm{P}-1(\# 2)$
2
$1.421 \mathrm{~g} / \mathrm{cm}^{3}$
956.00
$4.69 \mathrm{~cm}^{-1}$

B. Intensity Measurements

\section{Rigaku Saturn}

Rigaku AFC10

$\operatorname{MoK} \alpha(\lambda=0.71070 \AA)$

graphite monochromated

$70 \mathrm{~mm} \times 70 \mathrm{~mm}$

720 exposures

$-110.0-70.0^{\mathrm{O}}$

$30.0 \mathrm{sec} . /^{\mathrm{O}}$

$-20.42^{\mathrm{O}}$

$-110.0-70.0^{\mathrm{O}}$

$30.0 \mathrm{sec} . /^{\mathrm{O}}$

$-20.42^{\mathrm{O}}$

$44.84 \mathrm{~mm}$

$0.035 \mathrm{~mm}$

$55.0^{\circ}$

Total: 14967

Unique: $8838\left(\mathrm{R}_{\mathrm{int}}=0.061\right)$

Lorentz-polarization

Absorption

(trans. factors: $0.7721-1.0000$ ) 
C. Structure Solution and Refinement

Structure Solution

Refinement

Function Minimized

Least Squares Weights

Anomalous Dispersion

No. Observations $(\mathrm{I}>3.00 \Sigma(\mathrm{I}))$

No. Variables

Reflection/Parameter Ratio

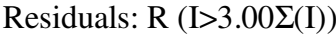

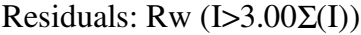

Goodness of Fit Indicator

Max Shift/Error in Final Cycle

Maximum peak in Final Diff. Map

Minimum peak in Final Diff. Map
Direct Methods (SIR92)

Full-matrix least-squares on $\mathrm{F}$

$\Sigma \mathrm{w}(|\mathrm{Fo}|-|\mathrm{Fc}|)^{2}$

$1 /\left[0.001 \mathrm{Fo}^{2}+3.000 \Sigma^{2}(\mathrm{Fo})+0.500\right]$

All non-hydrogen atoms

5174

595

8.70

0.063

0.090

0.95

0.01

$1.48 \mathrm{e}^{-/} \AA^{3}$

$-0.66 \mathrm{e}^{-} / \AA^{3}$ 
Table 1. Atomic coordinates and $\mathrm{B}_{\mathrm{iso}} / \mathrm{B}_{\mathrm{eq}}$

\begin{tabular}{|c|c|c|c|c|}
\hline atom & $\mathrm{x}$ & $\mathrm{y}$ & $\mathrm{z}$ & $\mathrm{B}_{\mathrm{eq}}$ \\
\hline $\mathrm{Fe}(1)$ & $0.81504(9)$ & $0.36858(8)$ & $0.93161(4)$ & $1.74(2)$ \\
\hline $\mathrm{P}(1)$ & $0.4436(2)$ & $-0.2637(1)$ & $0.74430(8)$ & $2.02(3)$ \\
\hline $\mathrm{F}(1)$ & $0.5118(4)$ & $-0.3697(4)$ & $0.7839(2)$ & $3.72(10)$ \\
\hline $\mathrm{F}(2)$ & $0.4419(4)$ & $-0.1781(3)$ & $0.8079(2)$ & $3.08(9)$ \\
\hline $\mathrm{F}(3)$ & $0.5895(4)$ & $-0.2133(3)$ & $0.7055(2)$ & $3.09(9)$ \\
\hline $\mathrm{F}(4)$ & $0.4445(5)$ & $-0.3493(3)$ & $0.6803(2)$ & $3.83(10)$ \\
\hline $\mathrm{F}(5)$ & $0.2985(4)$ & $-0.3143(3)$ & $0.7830(2)$ & $2.83(8)$ \\
\hline $\mathrm{F}(6)$ & $0.3751(4)$ & $-0.1582(4)$ & $0.7050(2)$ & $3.64(10)$ \\
\hline $\mathrm{O}(1)$ & $0.6046(4)$ & $0.1860(3)$ & $0.7038(2)$ & $1.80(8)$ \\
\hline $\mathrm{O}(2)$ & $0.7237(5)$ & $0.0462(4)$ & $0.8085(3)$ & $2.67(10)$ \\
\hline $\mathrm{O}(3)$ & $0.9554(5)$ & $-0.0493(5)$ & $0.8466(3)$ & $4.2(1)$ \\
\hline $\mathrm{O}(4)$ & $1.1847(5)$ & $0.0962(4)$ & $0.8499(2)$ & $2.81(10)$ \\
\hline $\mathrm{O}(5)$ & $1.2674(4)$ & $0.2942(3)$ & $0.7797(2)$ & $1.84(8)$ \\
\hline $\mathrm{O}(6)$ & $1.1456(4)$ & $0.4290(3)$ & $0.6750(2)$ & $1.90(8)$ \\
\hline $\mathrm{O}(7)$ & $0.9183(4)$ & $0.5260(3)$ & $0.6428(2)$ & $1.88(8)$ \\
\hline $\mathrm{O}(8)$ & $0.6902(4)$ & $0.3816(4)$ & $0.6330(2)$ & $2.13(9)$ \\
\hline $\mathrm{N}(1)$ & $0.9069(5)$ & $0.2689(4)$ & $0.7119(2)$ & $1.64(10)$ \\
\hline $\mathrm{C}(1)$ & $0.9300(6)$ & $0.2633(5)$ & $0.7858(3)$ & $1.54(11)$ \\
\hline$C(2)$ & $0.9817(6)$ & $0.1686(5)$ & $0.6707(3)$ & $1.80(12)$ \\
\hline $\mathrm{C}(3)$ & $0.9434(6)$ & $0.1544(5)$ & $0.6019(3)$ & $1.57(11)$ \\
\hline $\mathrm{C}(4)$ & $0.9828(7)$ & $0.2384(5)$ & $0.5461(3)$ & $2.14(13)$ \\
\hline$C(5)$ & $0.9428(7)$ & $0.2260(5)$ & $0.4830(3)$ & $2.08(13)$ \\
\hline$C(6)$ & $0.8671(6)$ & $0.1276(5)$ & $0.4732(3)$ & $2.00(12)$ \\
\hline$C(7)$ & $0.8281(8)$ & $0.1124(6)$ & $0.4037(3)$ & $3.0(2)$ \\
\hline $\mathrm{C}(8)$ & $0.8287(6)$ & $0.0426(5)$ & $0.5300(3)$ & $2.08(13)$ \\
\hline $\mathrm{C}(9)$ & $0.8649(6)$ & $0.0568(5)$ & $0.5933(3)$ & 1.91(12) \\
\hline$C(10)$ & $0.8769(6)$ & $0.3724(5)$ & $0.8255(3)$ & $1.62(11)$ \\
\hline $\mathrm{C}(11)$ & $0.7371(6)$ & $0.4089(5)$ & $0.8447(3)$ & $1.78(12)$ \\
\hline$C(12)$ & $0.7302(6)$ & $0.5135(5)$ & $0.8851(3)$ & $2.05(12)$ \\
\hline $\mathrm{C}(13)$ & $0.8610(7)$ & $0.5405(5)$ & $0.8920(3)$ & $2.36(13)$ \\
\hline $\mathrm{C}(14)$ & $0.9507(7)$ & $0.4547(5)$ & $0.8543(3)$ & $1.93(12)$ \\
\hline$C(15)$ & $0.7163(8)$ & $0.2205(6)$ & $0.9846(3)$ & $2.9(2)$ \\
\hline$C(16)$ & $0.6989(8)$ & $0.3201(6)$ & $1.0301(3)$ & $2.9(2)$ \\
\hline$C(17)$ & $0.8268(7)$ & $0.3568(6)$ & $1.0353(3)$ & $2.7(1)$ \\
\hline $\mathrm{C}(18)$ & $0.9261(8)$ & $0.2808(7)$ & $0.9948(3)$ & $3.2(2)$ \\
\hline $\mathrm{C}(19)$ & $0.8551(8)$ & $0.1975(6)$ & $0.9640(3)$ & $2.9(2)$ \\
\hline $\mathrm{C}(20)$ & $0.5904(7)$ & $0.3319(6)$ & $0.5341(3)$ & $2.5(1)$ \\
\hline
\end{tabular}


Table 1. Atomic coordinates and $\mathrm{B}_{\mathrm{iso}} / \mathrm{B}_{\mathrm{eq}}$ (continued)

\begin{tabular}{|c|c|c|c|c|}
\hline atom & $\mathrm{x}$ & $\mathrm{y}$ & $\mathrm{Z}$ & $\mathrm{B}_{\mathrm{eq}}$ \\
\hline $\mathrm{C}(21)$ & $0.5155(7)$ & $0.2518(7)$ & $0.5062(4)$ & $3.1(2)$ \\
\hline $\mathrm{C}(22)$ & $0.4691(7)$ & $0.1456(6)$ & $0.5439(4)$ & $2.8(2)$ \\
\hline $\mathrm{C}(23)$ & $0.4961(6)$ & $0.1189(6)$ & $0.6110(3)$ & $2.22(13)$ \\
\hline $\mathrm{C}(24)$ & $0.5710(6)$ & $0.2016(5)$ & $0.6389(3)$ & $2.09(12)$ \\
\hline$C(25)$ & $0.5407(6)$ & $0.0847(5)$ & $0.7485(3)$ & $1.90(12)$ \\
\hline$C(26)$ & $0.5882(6)$ & $0.0830(5)$ & $0.8167(3)$ & $2.14(13)$ \\
\hline$C(27)$ & $0.7390(7)$ & $-0.0775(6)$ & $0.8254(4)$ & $3.1(2)$ \\
\hline$C(28)$ & $0.8790(9)$ & $-0.1177(6)$ & $0.8146(5)$ & $4.2(2)$ \\
\hline C(29) & $1.0934(8)$ & $-0.0933(6)$ & $0.8345(5)$ & $4.2(2)$ \\
\hline$C(30)$ & $1.1582(8)$ & $-0.0241(6)$ & $0.8816(4)$ & $3.5(2)$ \\
\hline $\mathrm{C}(31)$ & $1.2509(6)$ & $0.1723(6)$ & $0.8839(3)$ & $2.21(13)$ \\
\hline$C(32)$ & $1.2736(7)$ & $0.1507(7)$ & $0.9510(4)$ & $3.2(2)$ \\
\hline $\mathrm{C}(33)$ & $1.3438(8)$ & $0.2353(7)$ & $0.9811(3)$ & $3.3(2)$ \\
\hline$C(34)$ & $1.3846(7)$ & $0.3423(6)$ & $0.9436(3)$ & $2.8(2)$ \\
\hline$C(35)$ & $1.3620(6)$ & $0.3655(6)$ & $0.8751(3)$ & $2.23(13)$ \\
\hline$C(36)$ & $1.2956(6)$ & $0.2815(5)$ & $0.8463(3)$ & $1.75(11)$ \\
\hline$C(37)$ & $1.3295(6)$ & $0.3950(5)$ & $0.7355(3)$ & $1.91(12)$ \\
\hline $\mathrm{C}(38)$ & $1.2845(6)$ & $0.3916(5)$ & $0.6670(3)$ & $1.98(12)$ \\
\hline C(39) & $1.1337(7)$ & $0.5577(5)$ & $0.6661(3)$ & $2.28(13)$ \\
\hline$C(40)$ & $0.9876(6)$ & $0.5907(5)$ & $0.6859(3)$ & $1.91(12)$ \\
\hline $\mathrm{C}(41)$ & $0.7839(6)$ & $0.5702(5)$ & $0.6486(3)$ & $2.16(13)$ \\
\hline$C(42)$ & $0.7197(7)$ & $0.5016(6)$ & $0.6007(4)$ & $2.8(2)$ \\
\hline $\mathrm{C}(43)$ & $0.6191(6)$ & $0.3081(5)$ & $0.6001(3)$ & $1.97(12)$ \\
\hline $\mathrm{H}(1)$ & $0.9372(5)$ & $0.3447(4)$ & $0.6892(2)$ & $2.02(13)$ \\
\hline $\mathrm{H}(2)$ & $0.8135(5)$ & $0.2608(4)$ & $0.7137(2)$ & $2.02(13)$ \\
\hline $\mathrm{H}(3)$ & $1.0241(6)$ & $0.2577(5)$ & $0.7832(3)$ & $1.9(1)$ \\
\hline $\mathrm{H}(4)$ & $0.8872(6)$ & $0.1932(5)$ & $0.8104(3)$ & $1.9(1)$ \\
\hline $\mathrm{H}(5)$ & $1.0752(6)$ & $0.1851(5)$ & $0.6621(3)$ & $2.2(1)$ \\
\hline $\mathrm{H}(6)$ & $0.9634(6)$ & $0.0948(5)$ & $0.6982(3)$ & $2.2(1)$ \\
\hline $\mathrm{H}(7)$ & $1.0370(7)$ & $0.3051(5)$ & $0.5511(3)$ & $2.6(2)$ \\
\hline $\mathrm{H}(8)$ & $0.9687(7)$ & $0.2854(5)$ & $0.4457(3)$ & $2.5(2)$ \\
\hline $\mathrm{H}(9)$ & $0.8616(8)$ & $0.1784(6)$ & $0.3719(3)$ & $3.9(2)$ \\
\hline $\mathrm{H}(10)$ & $0.7328(8)$ & $0.1104(6)$ & $0.4111(3)$ & $3.9(2)$ \\
\hline $\mathrm{H}(11)$ & $0.8649(8)$ & $0.0387(6)$ & $0.3844(3)$ & $3.9(2)$ \\
\hline $\mathrm{H}(12)$ & $0.7783(6)$ & $-0.0262(5)$ & $0.5240(3)$ & $2.6(2)$ \\
\hline $\mathrm{H}(13)$ & $0.8358(6)$ & $-0.0004(5)$ & $0.6315(3)$ & $2.2(1)$ \\
\hline $\mathrm{H}(14)$ & $0.6634(6)$ & $0.3696(5)$ & $0.8329(3)$ & $2.1(1)$ \\
\hline
\end{tabular}


Table1. Atomic coordinates and $\mathrm{B}_{\mathrm{iso}} / \mathrm{B}_{\mathrm{eq}}$ (continued)

\begin{tabular}{|c|c|c|c|c|}
\hline atom & $\mathrm{X}$ & $\mathrm{y}$ & $\mathrm{z}$ & $\mathrm{B}_{\mathrm{eq}}$ \\
\hline $\mathrm{H}(15)$ & $0.6502(6)$ & $0.5551(5)$ & $0.9061(3)$ & $2.5(2)$ \\
\hline $\mathrm{H}(16)$ & $0.8849(7)$ & $0.6051(5)$ & $0.9172(3)$ & $2.9(2)$ \\
\hline $\mathrm{H}(17)$ & $1.0454(7)$ & $0.4525(5)$ & $0.8500(3)$ & $2.4(2)$ \\
\hline $\mathrm{H}(18)$ & $0.6469(8)$ & $0.1773(6)$ & $0.9712(3)$ & $3.4(2)$ \\
\hline H(19) & $0.6156(8)$ & $0.3564(6)$ & $1.0512(3)$ & $3.3(2)$ \\
\hline $\mathrm{H}(20)$ & $0.8446(7)$ & $0.4225(6)$ & $1.0615(3)$ & $3.2(2)$ \\
\hline $\mathrm{H}(21)$ & $1.0207(8)$ & $0.2857(7)$ & $0.9897(3)$ & $3.9(2)$ \\
\hline $\mathrm{H}(22)$ & $0.8958(8)$ & $0.1359(6)$ & $0.9339(3)$ & $3.5(2)$ \\
\hline $\mathrm{H}(23)$ & $0.6215(7)$ & $0.4050(6)$ & $0.5082(3)$ & $3.0(2)$ \\
\hline $\mathrm{H}(24)$ & $0.4976(7)$ & $0.2683(7)$ & $0.4607(4)$ & $3.9(2)$ \\
\hline $\mathrm{H}(25)$ & $0.4180(7)$ & $0.0903(6)$ & $0.5247(4)$ & $3.5(2)$ \\
\hline $\mathrm{H}(26)$ & $0.4641(6)$ & $0.0466(6)$ & $0.6375(3)$ & $2.7(2)$ \\
\hline $\mathrm{H}(27)$ & $0.4456(6)$ & $0.0937(5)$ & $0.7573(3)$ & $2.3(1)$ \\
\hline $\mathrm{H}(28)$ & $0.5661(6)$ & $0.0109(5)$ & $0.7260(3)$ & $2.3(1)$ \\
\hline H(29) & $0.5333(6)$ & $0.0294(5)$ & $0.8502(3)$ & $2.6(2)$ \\
\hline $\mathrm{H}(30)$ & $0.5795(6)$ & $0.1627(5)$ & $0.8334(3)$ & $2.6(2)$ \\
\hline $\mathrm{H}(31)$ & $0.6950(7)$ & $-0.1223(6)$ & $0.7967(4)$ & $3.8(2)$ \\
\hline $\mathrm{H}(32)$ & $0.6975(7)$ & $-0.0932(6)$ & $0.8736(4)$ & $3.9(2)$ \\
\hline $\mathrm{H}(33)$ & $0.8808(9)$ & $-0.1992(6)$ & $0.8324(5)$ & $5.4(3)$ \\
\hline $\mathrm{H}(34)$ & $0.9162(9)$ & $-0.1138(6)$ & $0.7653(5)$ & $5.4(3)$ \\
\hline $\mathrm{H}(35)$ & $1.0967(8)$ & $-0.1780(6)$ & $0.8453(5)$ & $4.9(2)$ \\
\hline $\mathrm{H}(36)$ & $1.1377(8)$ & $-0.0766(6)$ & $0.7867(5)$ & $4.9(2)$ \\
\hline $\mathrm{H}(37)$ & $1.0981(8)$ & $-0.0198(6)$ & $0.9263(4)$ & $4.1(2)$ \\
\hline $\mathrm{H}(38)$ & $1.2395(8)$ & $-0.0624(6)$ & $0.8874(4)$ & $4.1(2)$ \\
\hline H(39) & $1.2429(7)$ & $0.0775(7)$ & $0.9768(4)$ & $3.6(2)$ \\
\hline $\mathrm{H}(40)$ & $1.3619(8)$ & $0.2197(7)$ & $1.0266(3)$ & $3.9(2)$ \\
\hline $\mathrm{H}(41)$ & $1.4291(7)$ & $0.4010(6)$ & $0.9641(3)$ & $3.4(2)$ \\
\hline $\mathrm{H}(42)$ & $1.3919(6)$ & $0.4390(6)$ & $0.8493(3)$ & $2.7(2)$ \\
\hline $\mathrm{H}(43)$ & $1.4246(6)$ & $0.3876(5)$ & $0.7274(3)$ & $2.3(1)$ \\
\hline $\mathrm{H}(44)$ & $1.3019(6)$ & $0.4690(5)$ & $0.7572(3)$ & $2.3(1)$ \\
\hline $\mathrm{H}(45)$ & $1.3393(6)$ & $0.4441(5)$ & $0.6327(3)$ & $2.4(2)$ \\
\hline $\mathrm{H}(46)$ & $1.2938(6)$ & $0.3110(5)$ & $0.6517(3)$ & $2.4(2)$ \\
\hline $\mathrm{H}(47)$ & $1.1814(7)$ & $0.5962(5)$ & $0.6956(3)$ & $2.8(2)$ \\
\hline $\mathrm{H}(48)$ & $1.1689(7)$ & $0.5830(5)$ & $0.6183(3)$ & $2.8(2)$ \\
\hline H(49) & $0.9759(6)$ & $0.6755(5)$ & $0.6787(3)$ & $2.3(2)$ \\
\hline $\mathrm{H}(50)$ & $0.9531(6)$ & $0.5678(5)$ & $0.7341(3)$ & $2.3(2)$ \\
\hline $\mathrm{H}(51)$ & $0.7853(6)$ & $0.6543(5)$ & $0.6356(3)$ & $2.6(2)$ \\
\hline $\mathrm{H}(52)$ & $0.7347(6)$ & $0.5577(5)$ & $0.6958(3)$ & $2.6(2)$ \\
\hline $\mathrm{H}(53)$ & $0.7800(7)$ & $0.4968(6)$ & $0.5561(4)$ & $3.3(2)$ \\
\hline $\mathrm{H}(54)$ & $0.6390(7)$ & $0.5410(6)$ & $0.5947(4)$ & $3.3(2)$ \\
\hline
\end{tabular}


Table 2. Anisotropic Displacement Parameters

\begin{tabular}{|c|c|c|c|c|c|c|}
\hline atom & $\mathrm{U}_{11}$ & $\mathrm{U}_{22}$ & $\mathrm{U}_{33}$ & $\mathrm{U}_{12}$ & $\mathrm{U}_{13}$ & $\mathrm{U}_{23}$ \\
\hline $\mathrm{Fe}(1)$ & $0.0245(5)$ & $0.0277(5)$ & $0.0145(4)$ & $0.0051(4)$ & $-0.0051(3)$ & $-0.0031(3)$ \\
\hline $\mathrm{P}(1)$ & $0.0302(9)$ & $0.0204(7)$ & $0.0273(8)$ & $-0.0022(6)$ & $-0.0088(7)$ & $-0.0024(6)$ \\
\hline $\mathrm{F}(1)$ & $0.048(3)$ & $0.037(2)$ & $0.055(3)$ & $0.012(2)$ & $-0.012(2)$ & $0.010(2)$ \\
\hline $\mathrm{F}(2)$ & $0.039(2)$ & $0.042(2)$ & $0.038(2)$ & $-0.011(2)$ & $-0.007(2)$ & $-0.015(2)$ \\
\hline $\mathrm{F}(3)$ & $0.032(2)$ & $0.031(2)$ & $0.051(2)$ & $-0.002(2)$ & $0.000(2)$ & $-0.006(2)$ \\
\hline $\mathrm{F}(4)$ & $0.071(3)$ & $0.039(2)$ & $0.033(2)$ & $-0.015(2)$ & $-0.001(2)$ & $-0.015(2)$ \\
\hline $\mathrm{F}(5)$ & $0.032(2)$ & $0.033(2)$ & $0.042(2)$ & $-0.009(2)$ & $-0.004(2)$ & $-0.007(2)$ \\
\hline $\mathrm{F}(6)$ & $0.045(3)$ & $0.040(2)$ & $0.057(3)$ & $0.000(2)$ & $-0.022(2)$ & $0.012(2)$ \\
\hline $\mathrm{O}(1)$ & $0.019(2)$ & $0.023(2)$ & $0.027(2)$ & $-0.001(2)$ & $-0.009(2)$ & $-0.001(2)$ \\
\hline $\mathrm{O}(2)$ & $0.022(2)$ & $0.021(2)$ & $0.058(3)$ & $-0.000(2)$ & $-0.011(2)$ & $0.008(2)$ \\
\hline $\mathrm{O}(3)$ & $0.034(3)$ & $0.040(3)$ & $0.087(4)$ & $0.003(2)$ & $-0.019(3)$ & $0.002(3)$ \\
\hline $\mathrm{O}(4)$ & $0.028(3)$ & $0.029(2)$ & $0.048(3)$ & $-0.002(2)$ & $-0.010(2)$ & $0.020(2)$ \\
\hline $\mathrm{O}(5)$ & $0.025(2)$ & $0.021(2)$ & $0.026(2)$ & $-0.002(2)$ & $-0.010(2)$ & $0.009(2)$ \\
\hline $\mathrm{O}(6)$ & $0.021(2)$ & $0.019(2)$ & $0.033(2)$ & $-0.002(2)$ & $-0.008(2)$ & $0.006(2)$ \\
\hline $\mathrm{O}(7)$ & $0.022(2)$ & $0.023(2)$ & $0.028(2)$ & $0.002(2)$ & $-0.008(2)$ & $-0.005(2)$ \\
\hline $\mathrm{O}(8)$ & $0.026(2)$ & $0.029(2)$ & $0.026(2)$ & $-0.003(2)$ & $-0.007(2)$ & $0.003(2)$ \\
\hline $\mathrm{N}(1)$ & $0.029(3)$ & $0.017(2)$ & $0.018(2)$ & $-0.001(2)$ & $-0.008(2)$ & $-0.003(2)$ \\
\hline $\mathrm{C}(1)$ & $0.022(3)$ & $0.020(3)$ & $0.017(3)$ & $-0.005(2)$ & $-0.007(2)$ & $0.001(2)$ \\
\hline$C(2)$ & $0.029(3)$ & $0.020(3)$ & $0.020(3)$ & $0.003(2)$ & $-0.005(2)$ & $-0.004(2)$ \\
\hline$C(3)$ & $0.021(3)$ & $0.022(3)$ & $0.018(3)$ & $0.001(2)$ & $-0.007(2)$ & $-0.004(2)$ \\
\hline C(4) & $0.035(4)$ & $0.025(3)$ & $0.022(3)$ & $-0.001(3)$ & $-0.007(3)$ & $-0.004(2)$ \\
\hline $\mathrm{C}(5)$ & $0.036(4)$ & $0.028(3)$ & $0.015(3)$ & $0.003(3)$ & $-0.005(2)$ & $0.000(2)$ \\
\hline$C(6)$ & $0.025(3)$ & $0.029(3)$ & $0.025(3)$ & $0.004(3)$ & $-0.013(3)$ & $-0.008(2)$ \\
\hline$C(7)$ & $0.046(5)$ & $0.043(4)$ & $0.032(4)$ & $0.005(3)$ & $-0.021(3)$ & $-0.009(3)$ \\
\hline $\mathrm{C}(8)$ & $0.029(4)$ & $0.024(3)$ & $0.029(3)$ & $-0.006(3)$ & $-0.011(3)$ & $-0.008(2)$ \\
\hline $\mathrm{C}(9)$ & $0.029(3)$ & $0.021(3)$ & $0.021(3)$ & $0.002(2)$ & $-0.001(2)$ & $-0.003(2)$ \\
\hline $\mathrm{C}(10)$ & $0.021(3)$ & $0.023(3)$ & $0.017(3)$ & $-0.004(2)$ & $-0.005(2)$ & $0.003(2)$ \\
\hline $\mathrm{C}(11)$ & $0.028(3)$ & $0.024(3)$ & $0.016(3)$ & $0.002(2)$ & $-0.007(2)$ & $0.001(2)$ \\
\hline $\mathrm{C}(12)$ & $0.028(4)$ & $0.027(3)$ & $0.024(3)$ & $0.010(3)$ & $-0.009(3)$ & $0.000(2)$ \\
\hline$C(13)$ & $0.045(4)$ & $0.023(3)$ & $0.023(3)$ & $-0.001(3)$ & $-0.011(3)$ & $-0.003(2)$ \\
\hline$C(14)$ & $0.030(4)$ & $0.026(3)$ & $0.019(3)$ & $-0.004(3)$ & $-0.008(3)$ & $-0.002(2)$ \\
\hline$C(15)$ & $0.048(5)$ & $0.034(4)$ & $0.025(3)$ & $0.002(3)$ & $-0.002(3)$ & $0.010(3)$ \\
\hline$C(16)$ & $0.040(4)$ & $0.050(4)$ & $0.016(3)$ & $0.010(3)$ & $0.003(3)$ & $-0.000(3)$ \\
\hline$C(17)$ & $0.039(4)$ & $0.050(4)$ & $0.013(3)$ & $0.008(3)$ & $-0.009(3)$ & $-0.004(3)$ \\
\hline C(18) & $0.045(5)$ & $0.056(4)$ & $0.021(3)$ & $0.021(4)$ & $-0.013(3)$ & $0.003(3)$ \\
\hline $\mathrm{C}(19)$ & $0.062(5)$ & $0.031(3)$ & $0.020(3)$ & $0.013(3)$ & $-0.012(3)$ & $0.001(3)$ \\
\hline $\mathrm{C}(20)$ & $0.028(4)$ & $0.042(4)$ & $0.026(3)$ & $0.003(3)$ & $-0.007(3)$ & $0.003(3)$ \\
\hline
\end{tabular}


Table 2. Anisotropic Displacement Parameters (continued)

$\begin{array}{lllllll}\text { atom } & \mathrm{U}_{11} & \mathrm{U}_{22} & \mathrm{U}_{33} & \mathrm{U}_{12} & \mathrm{U}_{13} & \mathrm{U}_{23} \\ \mathrm{C}(21) & 0.025(4) & 0.066(5) & 0.033(4) & 0.009(3) & -0.014(3) & -0.012(3) \\ \mathrm{C}(22) & 0.024(4) & 0.044(4) & 0.044(4) & 0.004(3) & -0.013(3) & -0.013(3) \\ \mathrm{C}(23) & 0.022(3) & 0.030(3) & 0.034(3) & 0.002(3) & -0.009(3) & -0.005(3) \\ \mathrm{C}(24) & 0.021(3) & 0.028(3) & 0.029(3) & 0.008(3) & -0.003(2) & -0.007(3) \\ \mathrm{C}(25) & 0.019(3) & 0.024(3) & 0.029(3) & -0.002(2) & -0.004(2) & 0.000(2) \\ \mathrm{C}(26) & 0.023(3) & 0.024(3) & 0.035(3) & -0.008(3) & -0.007(3) & 0.003(3) \\ \mathrm{C}(27) & 0.038(4) & 0.024(3) & 0.060(5) & -0.005(3) & -0.021(4) & 0.012(3) \\ \mathrm{C}(28) & 0.061(6) & 0.017(3) & 0.092(7) & -0.001(3) & -0.040(5) & 0.000(4) \\ \mathrm{C}(29) & 0.033(5) & 0.024(4) & 0.100(7) & 0.005(3) & -0.015(4) & 0.014(4) \\ \mathrm{C}(30) & 0.031(4) & 0.030(4) & 0.069(5) & 0.005(3) & -0.005(4) & 0.025(3) \\ \mathrm{C}(31) & 0.017(3) & 0.031(3) & 0.032(3) & 0.002(3) & 0.002(3) & 0.009(3) \\ \mathrm{C}(32) & 0.025(4) & 0.056(5) & 0.032(4) & 0.004(3) & 0.004(3) & 0.019(3) \\ \mathrm{C}(33) & 0.044(5) & 0.061(5) & 0.020(3) & 0.017(4) & -0.005(3) & 0.002(3) \\ \mathrm{C}(34) & 0.041(4) & 0.045(4) & 0.021(3) & 0.007(3) & -0.007(3) & -0.005(3) \\ \mathrm{C}(35) & 0.029(4) & 0.032(3) & 0.023(3) & 0.003(3) & -0.006(3) & -0.001(2) \\ \mathrm{C}(36) & 0.015(3) & 0.030(3) & 0.019(3) & 0.009(2) & -0.000(2) & 0.004(2) \\ \mathrm{C}(37) & 0.021(3) & 0.020(3) & 0.031(3) & -0.001(2) & -0.005(2) & 0.006(2) \\ \mathrm{C}(38) & 0.023(3) & 0.026(3) & 0.027(3) & -0.004(2) & -0.009(3) & -0.000(2) \\ \mathrm{C}(39) & 0.036(4) & 0.021(3) & 0.032(3) & -0.006(3) & -0.014(3) & 0.011(2) \\ \mathrm{C}(40) & 0.030(4) & 0.020(3) & 0.024(3) & 0.004(2) & -0.010(3) & -0.001(2) \\ \mathrm{C}(41) & 0.017(3) & 0.022(3) & 0.041(4) & 0.003(2) & -0.006(3) & 0.005(3) \\ \mathrm{C}(42) & 0.023(4) & 0.030(3) & 0.052(4) & 0.000(3) & -0.007(3) & 0.014(3) \\ \mathrm{C}(43) & 0.014(3) & 0.031(3) & 0.030(3) & 0.003(2) & -0.005(2) & -0.006(3) \\ & & & & & \end{array}$

The general temperature factor expression: $\exp \left(-2 \pi^{2}\left(\mathrm{a}^{*} \mathrm{U}_{11} \mathrm{~h}^{2}+\mathrm{b}^{* 2} \mathrm{U}_{22} \mathrm{k}^{2}+\mathrm{c}^{*} \mathrm{U}_{33} \mathrm{l}^{2}+2 \mathrm{a}^{*} \mathrm{~b} * \mathrm{U}_{12} \mathrm{hk}+\right.\right.$ $\left.\left.2 \mathrm{a} * \mathrm{c} * \mathrm{U}_{13} \mathrm{hl}+2 \mathrm{~b} * \mathrm{c} * \mathrm{U}_{23} \mathrm{kl}\right)\right)$ 
Table 3. Bond lengths ( $)$

\begin{tabular}{|c|c|c|c|c|c|}
\hline atom & atom & distance & atom & atom & distance \\
\hline $\mathrm{Fe}(1)$ & $C(10)$ & $2.021(5)$ & $\mathrm{Fe}(1)$ & $\mathrm{C}(11)$ & $2.046(6)$ \\
\hline $\mathrm{Fe}(1)$ & $\mathrm{C}(12)$ & $2.068(6)$ & $\mathrm{Fe}(1)$ & $\mathrm{C}(13)$ & $2.037(6)$ \\
\hline $\mathrm{Fe}(1)$ & $\mathrm{C}(14)$ & $2.017(6)$ & $\mathrm{Fe}(1)$ & $C(15)$ & $2.045(7)$ \\
\hline $\mathrm{Fe}(1)$ & $C(16)$ & $2.070(6)$ & $\mathrm{Fe}(1)$ & $\mathrm{C}(17)$ & $2.044(6)$ \\
\hline $\mathrm{Fe}(1)$ & $\mathrm{C}(18)$ & $2.045(6)$ & $\mathrm{Fe}(1)$ & $\mathrm{C}(19)$ & $2.025(6)$ \\
\hline $\mathrm{P}(1)$ & $\mathrm{F}(1)$ & $1.601(4)$ & $\mathrm{P}(1)$ & $\mathrm{F}(2)$ & $1.592(4)$ \\
\hline $\mathrm{P}(1)$ & $\mathrm{F}(3)$ & $1.607(4)$ & $\mathrm{P}(1)$ & $\mathrm{F}(4)$ & $1.601(4)$ \\
\hline $\mathrm{P}(1)$ & $\mathrm{F}(5)$ & $1.602(4)$ & $\mathrm{P}(1)$ & $\mathrm{F}(6)$ & $1.595(4)$ \\
\hline $\mathrm{O}(1)$ & $\mathrm{C}(24)$ & $1.382(7)$ & $\mathrm{O}(1)$ & $\mathrm{C}(25)$ & $1.454(7)$ \\
\hline $\mathrm{O}(2)$ & $\mathrm{C}(26)$ & $1.410(7)$ & $\mathrm{O}(2)$ & $\mathrm{C}(27)$ & $1.403(7)$ \\
\hline $\mathrm{O}(3)$ & $\mathrm{C}(28)$ & $1.359(9)$ & $\mathrm{O}(3)$ & C(29) & $1.453(9)$ \\
\hline $\mathrm{O}(4)$ & $\mathrm{C}(30)$ & $1.442(7)$ & $\mathrm{O}(4)$ & $\mathrm{C}(31)$ & $1.368(8)$ \\
\hline $\mathrm{O}(5)$ & $\mathrm{C}(36)$ & $1.387(6)$ & $\mathrm{O}(5)$ & $\mathrm{C}(37)$ & $1.439(6)$ \\
\hline $\mathrm{O}(6)$ & $\mathrm{C}(38)$ & $1.445(7)$ & $\mathrm{O}(6)$ & C(39) & $1.429(6)$ \\
\hline $\mathrm{O}(7)$ & $\mathrm{C}(40)$ & $1.434(7)$ & $\mathrm{O}(7)$ & $\mathrm{C}(41)$ & $1.429(7)$ \\
\hline $\mathrm{O}(8)$ & $\mathrm{C}(42)$ & $1.446(7)$ & $\mathrm{O}(8)$ & $\mathrm{C}(43)$ & $1.367(7)$ \\
\hline $\mathrm{N}(1)$ & $\mathrm{C}(1)$ & $1.505(6)$ & $\mathrm{N}(1)$ & $\mathrm{C}(2)$ & $1.499(7)$ \\
\hline $\mathrm{C}(1)$ & $\mathrm{C}(10)$ & $1.494(7)$ & $\mathrm{C}(2)$ & $C(3)$ & $1.492(7)$ \\
\hline$C(3)$ & $\mathrm{C}(4)$ & $1.386(8)$ & $C(3)$ & $\mathrm{C}(9)$ & $1.392(8)$ \\
\hline $\mathrm{C}(4)$ & $C(5)$ & $1.388(8)$ & $\mathrm{C}(5)$ & $C(6)$ & $1.389(8)$ \\
\hline$C(6)$ & $C(7)$ & $1.508(8)$ & $\mathrm{C}(6)$ & $\mathrm{C}(8)$ & $1.405(8)$ \\
\hline $\mathrm{C}(8)$ & $\mathrm{C}(9)$ & $1.378(8)$ & $C(10)$ & $\mathrm{C}(11)$ & $1.447(8)$ \\
\hline $\mathrm{C}(10)$ & $\mathrm{C}(14)$ & $1.402(8)$ & $\mathrm{C}(11)$ & $\mathrm{C}(12)$ & $1.424(8)$ \\
\hline $\mathrm{C}(12)$ & $\mathrm{C}(13)$ & $1.406(9)$ & $\mathrm{C}(13)$ & $\mathrm{C}(14)$ & $1.421(8)$ \\
\hline$C(15)$ & $C(16)$ & $1.431(9)$ & $C(15)$ & C(19) & $1.406(10)$ \\
\hline$C(16)$ & $\mathrm{C}(17)$ & $1.397(10)$ & $\mathrm{C}(17)$ & $\mathrm{C}(18)$ & $1.428(9)$ \\
\hline $\mathrm{C}(18)$ & C(19) & $1.414(10)$ & $\mathrm{C}(20)$ & $\mathrm{C}(21)$ & $1.384(9)$ \\
\hline$C(20)$ & $\mathrm{C}(43)$ & $1.391(8)$ & $\mathrm{C}(21)$ & $\mathrm{C}(22)$ & $1.385(10)$ \\
\hline $\mathrm{C}(22)$ & $\mathrm{C}(23)$ & $1.409(9)$ & $\mathrm{C}(23)$ & $\mathrm{C}(24)$ & $1.402(8)$ \\
\hline $\mathrm{C}(24)$ & $\mathrm{C}(43)$ & $1.400(8)$ & $\mathrm{C}(25)$ & $C(26)$ & $1.508(8)$ \\
\hline $\mathrm{C}(27)$ & $\mathrm{C}(28)$ & $1.460(10)$ & C(29) & $\mathrm{C}(30)$ & $1.489(11)$ \\
\hline $\mathrm{C}(31)$ & $\mathrm{C}(32)$ & $1.384(9)$ & $\mathrm{C}(31)$ & $C(36)$ & $1.408(8)$ \\
\hline $\mathrm{C}(32)$ & C(33) & $1.410(11)$ & C(33) & $\mathrm{C}(34)$ & $1.378(10)$ \\
\hline $\mathrm{C}(34)$ & $\mathrm{C}(35)$ & $1.413(8)$ & $\mathrm{C}(35)$ & $C(36)$ & $1.369(9)$ \\
\hline $\mathrm{C}(37)$ & C(38) & $1.503(8)$ & C(39) & $\mathrm{C}(40)$ & $1.497(9)$ \\
\hline $\mathrm{C}(41)$ & $\mathrm{C}(42)$ & $1.493(9)$ & & & \\
\hline
\end{tabular}


Table 4. Bond angles $\left({ }^{\mathrm{O}}\right)$

\begin{tabular}{|c|c|c|c|c|c|c|c|}
\hline atom & atom & atom & angle & atom & atom & atom & angle \\
\hline$C(10)$ & $\mathrm{Fe}(1)$ & $\mathrm{C}(11)$ & $41.7(2)$ & $C(10)$ & $\mathrm{Fe}(1)$ & $C(12)$ & $69.0(2)$ \\
\hline $\mathrm{C}(11)$ & $\mathrm{Fe}(1)$ & $\mathrm{C}(12)$ & $40.5(2)$ & $C(10)$ & $\mathrm{Fe}(1)$ & $\mathrm{C}(13)$ & $69.1(2)$ \\
\hline $\mathrm{C}(11)$ & $\mathrm{Fe}(1)$ & $\mathrm{C}(13)$ & $68.4(2)$ & $C(12)$ & $\mathrm{Fe}(1)$ & $\mathrm{C}(13)$ & $40.1(3)$ \\
\hline $\mathrm{C}(10)$ & $\mathrm{Fe}(1)$ & $\mathrm{C}(14)$ & $40.6(2)$ & $\mathrm{C}(11)$ & $\mathrm{Fe}(1)$ & $\mathrm{C}(14)$ & $68.7(2)$ \\
\hline $\mathrm{C}(12)$ & $\mathrm{Fe}(1)$ & $\mathrm{C}(14)$ & $68.1(3)$ & $\mathrm{C}(13)$ & $\mathrm{Fe}(1)$ & $\mathrm{C}(14)$ & $41.0(2)$ \\
\hline $\mathrm{C}(10)$ & $\mathrm{Fe}(1)$ & $C(15)$ & $120.2(3)$ & $\mathrm{C}(11)$ & $\mathrm{Fe}(1)$ & $\mathrm{C}(15)$ & $108.1(3)$ \\
\hline $\mathrm{C}(12)$ & $\mathrm{Fe}(1)$ & $C(15)$ & $126.6(3)$ & $\mathrm{C}(13)$ & $\mathrm{Fe}(1)$ & $\mathrm{C}(15)$ & $162.9(3)$ \\
\hline $\mathrm{C}(14)$ & $\mathrm{Fe}(1)$ & $C(15)$ & $154.9(2)$ & $C(10)$ & $\mathrm{Fe}(1)$ & $C(16)$ & $157.8(3)$ \\
\hline $\mathrm{C}(11)$ & $\mathrm{Fe}(1)$ & $C(16)$ & $123.7(3)$ & $C(12)$ & $\mathrm{Fe}(1)$ & $C(16)$ & 111.1(3) \\
\hline $\mathrm{C}(13)$ & $\mathrm{Fe}(1)$ & $C(16)$ & $126.2(3)$ & $\mathrm{C}(14)$ & $\mathrm{Fe}(1)$ & $C(16)$ & $161.4(3)$ \\
\hline $\mathrm{C}(10)$ & $\mathrm{Fe}(1)$ & $\mathrm{C}(17)$ & $158.9(3)$ & $\mathrm{C}(11)$ & $\mathrm{Fe}(1)$ & $\mathrm{C}(17)$ & $158.8(3)$ \\
\hline$C(12)$ & $\mathrm{Fe}(1)$ & $C(17)$ & $124.0(3)$ & $C(13)$ & $\mathrm{Fe}(1)$ & $\mathrm{C}(17)$ & $109.0(3)$ \\
\hline $\mathrm{C}(14)$ & $\mathrm{Fe}(1)$ & $C(17)$ & $124.2(3)$ & $C(10)$ & $\mathrm{Fe}(1)$ & $\mathrm{C}(18)$ & $120.7(3)$ \\
\hline $\mathrm{C}(11)$ & $\mathrm{Fe}(1)$ & $\mathrm{C}(18)$ & $159.1(2)$ & $C(12)$ & $\mathrm{Fe}(1)$ & $\mathrm{C}(18)$ & $157.6(3)$ \\
\hline $\mathrm{C}(13)$ & $\mathrm{Fe}(1)$ & $\mathrm{C}(18)$ & $120.9(3)$ & $\mathrm{C}(14)$ & $\mathrm{Fe}(1)$ & $\mathrm{C}(18)$ & $105.2(3)$ \\
\hline $\mathrm{C}(10)$ & $\mathrm{Fe}(1)$ & C(19) & $104.7(2)$ & $\mathrm{C}(11)$ & $\mathrm{Fe}(1)$ & C(19) & $123.5(3)$ \\
\hline$C(12)$ & $\mathrm{Fe}(1)$ & C(19) & $161.5(3)$ & $\mathrm{C}(13)$ & $\mathrm{Fe}(1)$ & C(19) & $155.6(3)$ \\
\hline $\mathrm{C}(14)$ & $\mathrm{Fe}(1)$ & C(19) & 119.1(3) & $C(15)$ & $\mathrm{Fe}(1)$ & $C(16)$ & $40.7(3)$ \\
\hline$C(15)$ & $\mathrm{Fe}(1)$ & $\mathrm{C}(17)$ & $67.8(3)$ & $C(16)$ & $\mathrm{Fe}(1)$ & $\mathrm{C}(17)$ & $39.7(3)$ \\
\hline$C(15)$ & $\mathrm{Fe}(1)$ & $\mathrm{C}(18)$ & $68.5(3)$ & $C(16)$ & $\mathrm{Fe}(1)$ & $\mathrm{C}(18)$ & $68.2(3)$ \\
\hline$C(17)$ & $\mathrm{Fe}(1)$ & $\mathrm{C}(18)$ & $40.9(3)$ & $C(15)$ & $\mathrm{Fe}(1)$ & C(19) & $40.4(3)$ \\
\hline$C(16)$ & $\mathrm{Fe}(1)$ & C(19) & $67.9(3)$ & $\mathrm{C}(17)$ & $\mathrm{Fe}(1)$ & C(19) & $67.9(3)$ \\
\hline $\mathrm{C}(18)$ & $\mathrm{Fe}(1)$ & C(19) & $40.6(3)$ & $\mathrm{F}(1)$ & $\mathrm{P}(1)$ & $\mathrm{F}(2)$ & $90.2(2)$ \\
\hline $\mathrm{F}(1)$ & $\mathrm{P}(1)$ & $\mathrm{F}(3)$ & $90.5(2)$ & $\mathrm{F}(2)$ & $\mathrm{P}(1)$ & $\mathrm{F}(3)$ & $90.2(2)$ \\
\hline $\mathrm{F}(1)$ & $\mathrm{P}(1)$ & $\mathrm{F}(4)$ & $90.2(2)$ & $\mathrm{F}(2)$ & $\mathrm{P}(1)$ & $F(4)$ & $179.6(3)$ \\
\hline $\mathrm{F}(3)$ & $\mathrm{P}(1)$ & $\mathrm{F}(4)$ & $90.0(2)$ & $\mathrm{F}(1)$ & $\mathrm{P}(1)$ & $F(5)$ & $89.3(2)$ \\
\hline $\mathrm{F}(2)$ & $\mathrm{P}(1)$ & $\mathrm{F}(5)$ & $89.9(2)$ & $\mathrm{F}(3)$ & $\mathrm{P}(1)$ & $F(5)$ & $179.8(2)$ \\
\hline $\mathrm{F}(4)$ & $\mathrm{P}(1)$ & $\mathrm{F}(5)$ & $89.9(2)$ & $\mathrm{F}(1)$ & $\mathrm{P}(1)$ & $F(6)$ & $179.8(3)$ \\
\hline $\mathrm{F}(2)$ & $\mathrm{P}(1)$ & $\mathrm{F}(6)$ & $89.8(2)$ & $\mathrm{F}(3)$ & $\mathrm{P}(1)$ & $F(6)$ & $89.7(2)$ \\
\hline $\mathrm{F}(4)$ & $\mathrm{P}(1)$ & $\mathrm{F}(6)$ & $89.9(2)$ & $\mathrm{F}(5)$ & $\mathrm{P}(1)$ & $\mathrm{F}(6)$ & $90.5(2)$ \\
\hline $\mathrm{C}(24)$ & $\mathrm{O}(1)$ & $\mathrm{C}(25)$ & $115.6(4)$ & $\mathrm{C}(26)$ & $\mathrm{O}(2)$ & $\mathrm{C}(27)$ & $113.3(5)$ \\
\hline $\mathrm{C}(28)$ & $\mathrm{O}(3)$ & C(29) & $111.4(6)$ & $\mathrm{C}(30)$ & $\mathrm{O}(4)$ & $\mathrm{C}(31)$ & $116.4(5)$ \\
\hline$C(36)$ & $\mathrm{O}(5)$ & $\mathrm{C}(37)$ & $116.2(4)$ & C(38) & $\mathrm{O}(6)$ & C(39) & $111.4(5)$ \\
\hline $\mathrm{C}(40)$ & $\mathrm{O}(7)$ & $\mathrm{C}(41)$ & $111.2(4)$ & $\mathrm{C}(42)$ & $\mathrm{O}(8)$ & $\mathrm{C}(43)$ & $116.2(5)$ \\
\hline $\mathrm{C}(1)$ & $\mathrm{N}(1)$ & $\mathrm{C}(2)$ & $110.5(4)$ & $\mathrm{N}(1)$ & $\mathrm{C}(1)$ & $C(10)$ & $112.8(4)$ \\
\hline $\mathrm{N}(1)$ & $\mathrm{C}(2)$ & $C(3)$ & $113.2(5)$ & $\mathrm{C}(2)$ & $C(3)$ & $\mathrm{C}(4)$ & $121.0(5)$ \\
\hline $\mathrm{C}(2)$ & $\mathrm{C}(3)$ & $\mathrm{C}(9)$ & $120.0(5)$ & $\mathrm{C}(4)$ & $C(3)$ & $\mathrm{C}(9)$ & $119.0(5)$ \\
\hline
\end{tabular}


Table 4. Bond angles $\left({ }^{\mathrm{O}}\right)$-- continued

\begin{tabular}{|c|c|c|c|c|c|c|c|}
\hline atom & atom & atom & angle & atom & atom & atom & angle \\
\hline $\mathrm{C}(3)$ & $\mathrm{C}(4)$ & $\mathrm{C}(5)$ & $120.3(6)$ & $\mathrm{C}(4)$ & $C(5)$ & $C(6)$ & $121.3(6)$ \\
\hline$C(5)$ & $\mathrm{C}(6)$ & $C(7)$ & $121.0(6)$ & $C(5)$ & $C(6)$ & $C(8)$ & $117.7(5)$ \\
\hline$C(7)$ & $C(6)$ & $\mathrm{C}(8)$ & $121.3(6)$ & $\mathrm{C}(6)$ & $\mathrm{C}(8)$ & $C(9)$ & $121.0(5)$ \\
\hline $\mathrm{C}(3)$ & $\mathrm{C}(9)$ & $\mathrm{C}(8)$ & $120.6(5)$ & $\mathrm{Fe}(1)$ & $C(10)$ & $\mathrm{C}(1)$ & $123.1(4)$ \\
\hline $\mathrm{Fe}(1)$ & $C(10)$ & $\mathrm{C}(11)$ & $70.1(3)$ & $\mathrm{C}(1)$ & $\mathrm{C}(10)$ & $\mathrm{C}(11)$ & $125.8(5)$ \\
\hline $\mathrm{Fe}(1)$ & $C(10)$ & $\mathrm{C}(14)$ & $69.5(3)$ & $\mathrm{C}(1)$ & $C(10)$ & $\mathrm{C}(14)$ & $127.0(5)$ \\
\hline $\mathrm{C}(11)$ & $\mathrm{C}(10)$ & $C(14)$ & $107.1(5)$ & $\mathrm{Fe}(1)$ & $\mathrm{C}(11)$ & $C(10)$ & $68.2(3)$ \\
\hline $\mathrm{Fe}(1)$ & $\mathrm{C}(11)$ & $C(12)$ & $70.6(3)$ & $C(10)$ & $\mathrm{C}(11)$ & $C(12)$ & $107.5(5)$ \\
\hline $\mathrm{Fe}(1)$ & $\mathrm{C}(12)$ & $\mathrm{C}(11)$ & $68.9(3)$ & $\mathrm{Fe}(1)$ & $\mathrm{C}(12)$ & $\mathrm{C}(13)$ & $68.8(3)$ \\
\hline $\mathrm{C}(11)$ & $\mathrm{C}(12)$ & $\mathrm{C}(13)$ & $108.3(5)$ & $\mathrm{Fe}(1)$ & $\mathrm{C}(13)$ & $\mathrm{C}(12)$ & $71.2(4)$ \\
\hline $\mathrm{Fe}(1)$ & $\mathrm{C}(13)$ & $\mathrm{C}(14)$ & $68.8(3)$ & $C(12)$ & $\mathrm{C}(13)$ & $\mathrm{C}(14)$ & $108.0(5)$ \\
\hline $\mathrm{Fe}(1)$ & $\mathrm{C}(14)$ & $C(10)$ & $69.8(3)$ & $\mathrm{Fe}(1)$ & $\mathrm{C}(14)$ & $\mathrm{C}(13)$ & $70.2(4)$ \\
\hline$C(10)$ & $\mathrm{C}(14)$ & $\mathrm{C}(13)$ & 109.1(6) & $\mathrm{Fe}(1)$ & $C(15)$ & $C(16)$ & $70.6(4)$ \\
\hline $\mathrm{Fe}(1)$ & $\mathrm{C}(15)$ & $C(19)$ & $69.0(4)$ & $C(16)$ & $C(15)$ & C(19) & $107.5(7)$ \\
\hline $\mathrm{Fe}(1)$ & $C(16)$ & $C(15)$ & $68.7(3)$ & $\mathrm{Fe}(1)$ & $C(16)$ & $\mathrm{C}(17)$ & $69.2(4)$ \\
\hline$C(15)$ & $C(16)$ & $\mathrm{C}(17)$ & $107.5(6)$ & $\mathrm{Fe}(1)$ & $\mathrm{C}(17)$ & $C(16)$ & 71.1(4) \\
\hline $\mathrm{Fe}(1)$ & $\mathrm{C}(17)$ & $\mathrm{C}(18)$ & $69.6(3)$ & $C(16)$ & $C(17)$ & $\mathrm{C}(18)$ & $109.4(6)$ \\
\hline $\mathrm{Fe}(1)$ & $\mathrm{C}(18)$ & $C(17)$ & $69.5(4)$ & $\mathrm{Fe}(1)$ & $\mathrm{C}(18)$ & C(19) & $68.9(4)$ \\
\hline $\mathrm{C}(17)$ & $\mathrm{C}(18)$ & $C(19)$ & $106.2(7)$ & $\mathrm{Fe}(1)$ & $C(19)$ & $C(15)$ & $70.6(4)$ \\
\hline $\mathrm{Fe}(1)$ & C(19) & $\mathrm{C}(18)$ & $70.4(4)$ & $C(15)$ & $C(19)$ & $\mathrm{C}(18)$ & $109.4(6)$ \\
\hline $\mathrm{C}(21)$ & $\mathrm{C}(20)$ & $\mathrm{C}(43)$ & $120.9(6)$ & $\mathrm{C}(20)$ & $\mathrm{C}(21)$ & $\mathrm{C}(22)$ & $119.9(6)$ \\
\hline $\mathrm{C}(21)$ & $\mathrm{C}(22)$ & $\mathrm{C}(23)$ & $120.6(6)$ & $\mathrm{C}(22)$ & $\mathrm{C}(23)$ & $\mathrm{C}(24)$ & $118.8(6)$ \\
\hline $\mathrm{O}(1)$ & $\mathrm{C}(24)$ & $\mathrm{C}(23)$ & $124.5(6)$ & $\mathrm{O}(1)$ & $\mathrm{C}(24)$ & $\mathrm{C}(43)$ & $115.1(5)$ \\
\hline $\mathrm{C}(23)$ & $\mathrm{C}(24)$ & $\mathrm{C}(43)$ & $120.4(6)$ & $\mathrm{O}(1)$ & $C(25)$ & $C(26)$ & $108.0(5)$ \\
\hline $\mathrm{O}(2)$ & $C(26)$ & $\mathrm{C}(25)$ & $112.6(5)$ & $\mathrm{O}(2)$ & $\mathrm{C}(27)$ & $\mathrm{C}(28)$ & $113.9(6)$ \\
\hline $\mathrm{O}(3)$ & $\mathrm{C}(28)$ & $\mathrm{C}(27)$ & $113.6(6)$ & $\mathrm{O}(3)$ & C(29) & $\mathrm{C}(30)$ & $105.3(6)$ \\
\hline $\mathrm{O}(4)$ & $\mathrm{C}(30)$ & C(29) & $107.6(6)$ & $\mathrm{O}(4)$ & $\mathrm{C}(31)$ & $\mathrm{C}(32)$ & $125.3(6)$ \\
\hline $\mathrm{O}(4)$ & $\mathrm{C}(31)$ & $C(36)$ & $115.4(5)$ & $\mathrm{C}(32)$ & $\mathrm{C}(31)$ & $C(36)$ & $119.3(6)$ \\
\hline $\mathrm{C}(31)$ & $C(32)$ & $\mathrm{C}(33)$ & $120.7(6)$ & $\mathrm{C}(32)$ & C(33) & $\mathrm{C}(34)$ & $118.8(6)$ \\
\hline $\mathrm{C}(33)$ & $\mathrm{C}(34)$ & $\mathrm{C}(35)$ & $121.0(7)$ & $\mathrm{C}(34)$ & $\mathrm{C}(35)$ & $\mathrm{C}(36)$ & $119.3(6)$ \\
\hline $\mathrm{O}(5)$ & $C(36)$ & $\mathrm{C}(31)$ & $114.7(5)$ & $\mathrm{O}(5)$ & $\mathrm{C}(36)$ & $\mathrm{C}(35)$ & $124.6(5)$ \\
\hline $\mathrm{C}(31)$ & $C(36)$ & $\mathrm{C}(35)$ & $120.8(5)$ & $\mathrm{O}(5)$ & C(37) & $\mathrm{C}(38)$ & $107.1(5)$ \\
\hline $\mathrm{O}(6)$ & $\mathrm{C}(38)$ & $\mathrm{C}(37)$ & $111.4(5)$ & $\mathrm{O}(6)$ & C(39) & $\mathrm{C}(40)$ & $108.2(5)$ \\
\hline $\mathrm{O}(7)$ & $\mathrm{C}(40)$ & C(39) & $108.0(5)$ & $\mathrm{O}(7)$ & $\mathrm{C}(41)$ & $\mathrm{C}(42)$ & $108.2(5)$ \\
\hline $\mathrm{O}(8)$ & $C(42)$ & $\mathrm{C}(41)$ & $107.4(5)$ & $\mathrm{O}(8)$ & $\mathrm{C}(43)$ & $\mathrm{C}(20)$ & $125.7(6)$ \\
\hline $\mathrm{O}(8)$ & $C(43)$ & $\mathrm{C}(24)$ & $114.9(5)$ & $\mathrm{C}(20)$ & C(43) & $\mathrm{C}(24)$ & $119.4(6)$ \\
\hline
\end{tabular}


Table 5. Bond angles involving hydrogens $\left({ }^{\circ}\right)$

\begin{tabular}{|c|c|c|c|c|c|c|c|}
\hline atom & atom & atom & angle & atom & atom & atom & angle \\
\hline $\mathrm{C}(1)$ & $\mathrm{N}(1)$ & $\mathrm{H}(1)$ & $109.3(5)$ & $\mathrm{C}(2)$ & $\mathrm{N}(1)$ & $\mathrm{H}(1)$ & $109.3(5)$ \\
\hline $\mathrm{C}(1)$ & $\mathrm{N}(1)$ & $\mathrm{H}(2)$ & $109.2(5)$ & $\mathrm{C}(2)$ & $\mathrm{N}(1)$ & $\mathrm{H}(2)$ & $109.0(5)$ \\
\hline $\mathrm{H}(1)$ & $\mathrm{N}(1)$ & $\mathrm{H}(2)$ & $109.5(6)$ & $\mathrm{N}(1)$ & $\mathrm{C}(1)$ & $\mathrm{H}(3)$ & $108.4(6)$ \\
\hline $\mathrm{C}(10)$ & $C(1)$ & $\mathrm{H}(3)$ & $108.4(6)$ & $\mathrm{N}(1)$ & $\mathrm{C}(1)$ & $\mathrm{H}(4)$ & $108.8(6)$ \\
\hline$C(10)$ & $\mathrm{C}(1)$ & $\mathrm{H}(4)$ & $108.9(6)$ & $\mathrm{H}(3)$ & $\mathrm{C}(1)$ & $\mathrm{H}(4)$ & $109.5(7)$ \\
\hline $\mathrm{N}(1)$ & $C(2)$ & $\mathrm{H}(5)$ & $108.4(6)$ & $\mathrm{C}(3)$ & $\mathrm{C}(2)$ & $\mathrm{H}(5)$ & $108.7(6)$ \\
\hline $\mathrm{N}(1)$ & $\mathrm{C}(2)$ & $\mathrm{H}(6)$ & $108.4(6)$ & $C(3)$ & $\mathrm{C}(2)$ & $\mathrm{H}(6)$ & $108.5(6)$ \\
\hline $\mathrm{H}(5)$ & $\mathrm{C}(2)$ & $\mathrm{H}(6)$ & $109.5(8)$ & $C(3)$ & $\mathrm{C}(4)$ & $\mathrm{H}(7)$ & $119.8(7)$ \\
\hline$C(5)$ & $\mathrm{C}(4)$ & $\mathrm{H}(7)$ & $119.8(7)$ & $\mathrm{C}(4)$ & $C(5)$ & $\mathrm{H}(8)$ & $119.4(7)$ \\
\hline$C(6)$ & $\mathrm{C}(5)$ & $\mathrm{H}(8)$ & 119.3(7) & $\mathrm{C}(6)$ & $\mathrm{C}(7)$ & $\mathrm{H}(9)$ & $109.6(8)$ \\
\hline$C(6)$ & $\mathrm{C}(7)$ & $\mathrm{H}(10)$ & 109.2(8) & $\mathrm{H}(9)$ & $C(7)$ & $\mathrm{H}(10)$ & $109.5(9)$ \\
\hline$C(6)$ & $\mathrm{C}(7)$ & $\mathrm{H}(11)$ & $109.6(8)$ & $\mathrm{H}(9)$ & $C(7)$ & $\mathrm{H}(11)$ & $109.5(9)$ \\
\hline $\mathrm{H}(10)$ & $C(7)$ & $\mathrm{H}(11)$ & $109.5(9)$ & $\mathrm{C}(6)$ & $\mathrm{C}(8)$ & $\mathrm{H}(12)$ & $119.0(7)$ \\
\hline $\mathrm{C}(9)$ & $\mathrm{C}(8)$ & $\mathrm{H}(12)$ & $120.0(7)$ & $\mathrm{C}(3)$ & $\mathrm{C}(9)$ & $\mathrm{H}(13)$ & $119.7(7)$ \\
\hline $\mathrm{C}(8)$ & $\mathrm{C}(9)$ & $\mathrm{H}(13)$ & 119.7(7) & $\mathrm{Fe}(1)$ & $\mathrm{C}(11)$ & $\mathrm{H}(14)$ & $126.0(6)$ \\
\hline $\mathrm{C}(10)$ & $\mathrm{C}(11)$ & $\mathrm{H}(14)$ & $126.2(7)$ & $\mathrm{C}(12)$ & $\mathrm{C}(11)$ & $\mathrm{H}(14)$ & $126.3(7)$ \\
\hline $\mathrm{Fe}(1)$ & $\mathrm{C}(12)$ & $\mathrm{H}(15)$ & $125.4(6)$ & $\mathrm{C}(11)$ & $\mathrm{C}(12)$ & $\mathrm{H}(15)$ & $125.7(7)$ \\
\hline$C(13)$ & $\mathrm{C}(12)$ & $\mathrm{H}(15)$ & $125.9(7)$ & $\mathrm{Fe}(1)$ & $\mathrm{C}(13)$ & $\mathrm{H}(16)$ & $125.9(6)$ \\
\hline$C(12)$ & $\mathrm{C}(13)$ & $\mathrm{H}(16)$ & $125.8(7)$ & $\mathrm{C}(14)$ & $\mathrm{C}(13)$ & $\mathrm{H}(16)$ & $126.2(7)$ \\
\hline $\mathrm{Fe}(1)$ & $\mathrm{C}(14)$ & $\mathrm{H}(17)$ & $125.3(6)$ & $\mathrm{C}(10)$ & $\mathrm{C}(14)$ & $\mathrm{H}(17)$ & $125.3(7)$ \\
\hline $\mathrm{C}(13)$ & $\mathrm{C}(14)$ & $\mathrm{H}(17)$ & $125.6(7)$ & $\mathrm{Fe}(1)$ & $\mathrm{C}(15)$ & $\mathrm{H}(18)$ & $126.1(7)$ \\
\hline$C(16)$ & $C(15)$ & $\mathrm{H}(18)$ & $126.4(8)$ & C(19) & $\mathrm{C}(15)$ & $\mathrm{H}(18)$ & $126.1(8)$ \\
\hline $\mathrm{Fe}(1)$ & $C(16)$ & $\mathrm{H}(19)$ & $125.6(7)$ & $C(15)$ & $\mathrm{C}(16)$ & H(19) & $126.0(8)$ \\
\hline $\mathrm{C}(17)$ & $C(16)$ & $\mathrm{H}(19)$ & $126.5(8)$ & $\mathrm{Fe}(1)$ & $\mathrm{C}(17)$ & $\mathrm{H}(20)$ & $125.3(7)$ \\
\hline$C(16)$ & $\mathrm{C}(17)$ & $\mathrm{H}(20)$ & $125.1(8)$ & $\mathrm{C}(18)$ & $\mathrm{C}(17)$ & $\mathrm{H}(20)$ & $125.5(8)$ \\
\hline $\mathrm{Fe}(1)$ & $\mathrm{C}(18)$ & $\mathrm{H}(21)$ & $126.2(7)$ & $\mathrm{C}(17)$ & $\mathrm{C}(18)$ & $\mathrm{H}(21)$ & $126.6(8)$ \\
\hline$C(19)$ & $\mathrm{C}(18)$ & $\mathrm{H}(21)$ & $127.2(8)$ & $\mathrm{Fe}(1)$ & C(19) & $\mathrm{H}(22)$ & $125.2(7)$ \\
\hline$C(15)$ & $\mathrm{C}(19)$ & $\mathrm{H}(22)$ & $125.7(8)$ & $\mathrm{C}(18)$ & C(19) & $\mathrm{H}(22)$ & $124.8(8)$ \\
\hline $\mathrm{C}(21)$ & $\mathrm{C}(20)$ & $\mathrm{H}(23)$ & $119.8(8)$ & $\mathrm{C}(43)$ & $\mathrm{C}(20)$ & $\mathrm{H}(23)$ & $119.3(8)$ \\
\hline$C(20)$ & $\mathrm{C}(21)$ & $\mathrm{H}(24)$ & $120.2(8)$ & $\mathrm{C}(22)$ & $\mathrm{C}(21)$ & $\mathrm{H}(24)$ & $119.8(8)$ \\
\hline $\mathrm{C}(21)$ & $\mathrm{C}(22)$ & $\mathrm{H}(25)$ & $120.0(8)$ & $\mathrm{C}(23)$ & $\mathrm{C}(22)$ & $\mathrm{H}(25)$ & $119.5(8)$ \\
\hline$C(22)$ & $\mathrm{C}(23)$ & $\mathrm{H}(26)$ & 121.1(7) & $\mathrm{C}(24)$ & $\mathrm{C}(23)$ & $\mathrm{H}(26)$ & $120.0(7)$ \\
\hline $\mathrm{O}(1)$ & $C(25)$ & $\mathrm{H}(27)$ & $110.0(6)$ & $C(26)$ & $\mathrm{C}(25)$ & $\mathrm{H}(27)$ & $110.3(6)$ \\
\hline $\mathrm{O}(1)$ & $C(25)$ & $\mathrm{H}(28)$ & $109.8(6)$ & $C(26)$ & $\mathrm{C}(25)$ & $\mathrm{H}(28)$ & $109.3(6)$ \\
\hline $\mathrm{H}(27)$ & $C(25)$ & $\mathrm{H}(28)$ & $109.5(8)$ & $\mathrm{O}(2)$ & $C(26)$ & H(29) & $109.0(7)$ \\
\hline$C(25)$ & $C(26)$ & H(29) & 109.1(7) & $\mathrm{O}(2)$ & $C(26)$ & $\mathrm{H}(30)$ & $108.4(7)$ \\
\hline$C(25)$ & $C(26)$ & $\mathrm{H}(30)$ & $108.2(7)$ & H(29) & $C(26)$ & $\mathrm{H}(30)$ & $109.5(8)$ \\
\hline
\end{tabular}


Table 5. Bond angles involving hydrogens $\left({ }^{\circ}\right)$-- continued

\begin{tabular}{|c|c|c|c|c|c|c|c|}
\hline atom & atom & atom & angle & atom & atom & atom & angle \\
\hline $\mathrm{O}(2)$ & $\mathrm{C}(27)$ & $\mathrm{H}(31)$ & $108.0(8)$ & $\mathrm{C}(28)$ & $C(27)$ & $\mathrm{H}(31)$ & $108.7(8)$ \\
\hline $\mathrm{O}(2)$ & $\mathrm{C}(27)$ & $\mathrm{H}(32)$ & $108.5(8)$ & $\mathrm{C}(28)$ & $C(27)$ & $\mathrm{H}(32)$ & $108.2(8)$ \\
\hline $\mathrm{H}(31)$ & $\mathrm{C}(27)$ & $\mathrm{H}(32)$ & $109.5(9)$ & $\mathrm{O}(3)$ & $\mathrm{C}(28)$ & $\mathrm{H}(33)$ & 108.7(9) \\
\hline $\mathrm{C}(27)$ & $\mathrm{C}(28)$ & $\mathrm{H}(33)$ & $108.5(9)$ & $\mathrm{O}(3)$ & $\mathrm{C}(28)$ & $\mathrm{H}(34)$ & 108.6(9) \\
\hline $\mathrm{C}(27)$ & $\mathrm{C}(28)$ & $\mathrm{H}(34)$ & 107.9(9) & $\mathrm{H}(33)$ & $\mathrm{C}(28)$ & $\mathrm{H}(34)$ & $109.5(11)$ \\
\hline $\mathrm{O}(3)$ & C(29) & $\mathrm{H}(35)$ & $111.2(9)$ & $\mathrm{C}(30)$ & $C(29)$ & $\mathrm{H}(35)$ & 111.1(9) \\
\hline $\mathrm{O}(3)$ & C(29) & $\mathrm{H}(36)$ & $110.2(9)$ & $\mathrm{C}(30)$ & $C(29)$ & $\mathrm{H}(36)$ & $109.6(9)$ \\
\hline $\mathrm{H}(35)$ & C(29) & $\mathrm{H}(36)$ & $109.5(11)$ & $\mathrm{O}(4)$ & $C(30)$ & $\mathrm{H}(37)$ & $109.7(8)$ \\
\hline $\mathrm{C}(29)$ & $\mathrm{C}(30)$ & $\mathrm{H}(37)$ & $109.0(8)$ & $\mathrm{O}(4)$ & $C(30)$ & $\mathrm{H}(38)$ & $110.3(8)$ \\
\hline $\mathrm{C}(29)$ & $C(30)$ & $\mathrm{H}(38)$ & $110.7(8)$ & $\mathrm{H}(37)$ & $C(30)$ & $\mathrm{H}(38)$ & $109.5(10)$ \\
\hline $\mathrm{C}(31)$ & $C(32)$ & $\mathrm{H}(39)$ & $119.7(8)$ & $\mathrm{C}(33)$ & $C(32)$ & $\mathrm{H}(39)$ & $119.6(8)$ \\
\hline$C(32)$ & $\mathrm{C}(33)$ & $\mathrm{H}(40)$ & $121.0(8)$ & $\mathrm{C}(34)$ & $C(33)$ & $\mathrm{H}(40)$ & $120.2(8)$ \\
\hline $\mathrm{C}(33)$ & $\mathrm{C}(34)$ & $\mathrm{H}(41)$ & $119.4(8)$ & $\mathrm{C}(35)$ & $C(34)$ & $\mathrm{H}(41)$ & $119.6(8)$ \\
\hline $\mathrm{C}(34)$ & $\mathrm{C}(35)$ & $\mathrm{H}(42)$ & $120.4(7)$ & $C(36)$ & $C(35)$ & $\mathrm{H}(42)$ & $120.3(7)$ \\
\hline $\mathrm{O}(5)$ & $\mathrm{C}(37)$ & $\mathrm{H}(43)$ & $110.0(6)$ & $\mathrm{C}(38)$ & $C(37)$ & $\mathrm{H}(43)$ & $110.2(6)$ \\
\hline $\mathrm{O}(5)$ & $\mathrm{C}(37)$ & $\mathrm{H}(44)$ & $110.2(6)$ & $\mathrm{C}(38)$ & $C(37)$ & $\mathrm{H}(44)$ & $109.9(6)$ \\
\hline $\mathrm{H}(43)$ & $C(37)$ & $\mathrm{H}(44)$ & $109.5(8)$ & $\mathrm{O}(6)$ & $\mathrm{C}(38)$ & $\mathrm{H}(45)$ & $109.0(6)$ \\
\hline $\mathrm{C}(37)$ & $\mathrm{C}(38)$ & $\mathrm{H}(45)$ & $109.4(6)$ & $\mathrm{O}(6)$ & $\mathrm{C}(38)$ & $\mathrm{H}(46)$ & $108.9(6)$ \\
\hline$C(37)$ & $\mathrm{C}(38)$ & $\mathrm{H}(46)$ & $108.6(6)$ & $\mathrm{H}(45)$ & $\mathrm{C}(38)$ & $\mathrm{H}(46)$ & $109.5(8)$ \\
\hline $\mathrm{O}(6)$ & C(39) & $\mathrm{H}(47)$ & $109.8(7)$ & $\mathrm{C}(40)$ & $C(39)$ & $\mathrm{H}(47)$ & $109.8(7)$ \\
\hline $\mathrm{O}(6)$ & C(39) & $\mathrm{H}(48)$ & $110.0(7)$ & $\mathrm{C}(40)$ & $C(39)$ & $\mathrm{H}(48)$ & $109.6(7)$ \\
\hline $\mathrm{H}(47)$ & $\mathrm{C}(39)$ & $\mathrm{H}(48)$ & $109.5(8)$ & $\mathrm{O}(7)$ & $\mathrm{C}(40)$ & $\mathrm{H}(49)$ & $110.1(6)$ \\
\hline C(39) & $\mathrm{C}(40)$ & $\mathrm{H}(49)$ & $110.3(7)$ & $\mathrm{O}(7)$ & $\mathrm{C}(40)$ & $\mathrm{H}(50)$ & $109.5(6)$ \\
\hline $\mathrm{C}(39)$ & $\mathrm{C}(40)$ & $\mathrm{H}(50)$ & $109.5(6)$ & $\mathrm{H}(49)$ & $\mathrm{C}(40)$ & $\mathrm{H}(50)$ & $109.5(8)$ \\
\hline $\mathrm{O}(7)$ & $\mathrm{C}(41)$ & $\mathrm{H}(51)$ & $109.8(7)$ & $\mathrm{C}(42)$ & $\mathrm{C}(41)$ & $\mathrm{H}(51)$ & $110.2(7)$ \\
\hline $\mathrm{O}(7)$ & $\mathrm{C}(41)$ & $\mathrm{H}(52)$ & $109.6(7)$ & $\mathrm{C}(42)$ & $\mathrm{C}(41)$ & $\mathrm{H}(52)$ & $109.5(7)$ \\
\hline $\mathrm{H}(51)$ & $\mathrm{C}(41)$ & $\mathrm{H}(52)$ & $109.5(8)$ & $\mathrm{O}(8)$ & $C(42)$ & $\mathrm{H}(53)$ & $110.2(7)$ \\
\hline$C(41)$ & $\mathrm{C}(42)$ & $\mathrm{H}(53)$ & $109.6(7)$ & $\mathrm{O}(8)$ & $\mathrm{C}(42)$ & $\mathrm{H}(54)$ & $110.0(7)$ \\
\hline$C(41)$ & $\mathrm{C}(42)$ & $\mathrm{H}(54)$ & $110.2(7)$ & $\mathrm{H}(53)$ & $\mathrm{C}(42)$ & $\mathrm{H}(54)$ & $109.5(9)$ \\
\hline
\end{tabular}


Table 6. Least Squares Planes

Plane number 1

Co-ordinates of the defining atoms projected onto the best plane

$\begin{array}{lllll}\text { Type } & \text { Serial } & \text { XP } & \text { YP } & \text { ZP } \\ \text { C } & 3 & -1.399 & 0.027 & 0.002 \\ \text { C } & 4 & -0.675 & 1.209 & 0.010 \\ \text { C } & 5 & 0.713 & 1.180 & -0.013 \\ \text { C } & 6 & 1.411 & -0.021 & 0.005 \\ \text { C } & 8 & 0.663 & -1.211 & 0.007 \\ \text { C } & 9 & -0.714 & -1.184 & -0.010\end{array}$

Plane number 2

Co-ordinates of the defining atoms projected onto the best plane

$\begin{array}{lllll}\text { Type } & \text { Serial } & \text { XP } & \text { YP } & \text { ZP } \\ \text { C } & 20 & 0.964 & 0.996 & 0.001 \\ \text { C } & 21 & 1.353 & -0.332 & -0.005 \\ \text { C } & 22 & 0.396 & -1.333 & 0.003 \\ \text { C } & 23 & -0.976 & -1.013 & 0.001 \\ \text { C } & 24 & -1.354 & 0.337 & -0.005 \\ \text { C } & 43 & -0.383 & 1.346 & 0.004\end{array}$

Plane number 3

Co-ordinates of the defining atoms projected onto the best plane

$\begin{array}{lllll}\text { Type } & \text { Serial } & \text { XP } & \text { YP } & \text { ZP } \\ \text { C } & 31 & 0.430 & -1.329 & 0.002 \\ \text { C } & 32 & -0.923 & -1.038 & -0.010 \\ \text { C } & 33 & -1.372 & 0.298 & 0.013 \\ \text { C } & 34 & -0.440 & 1.313 & -0.009 \\ \text { C } & 35 & 0.944 & 1.030 & 0.002 \\ \text { C } & 36 & 1.362 & -0.274 & 0.002\end{array}$

Plane number 4

Co-ordinates of the defining atoms projected onto the best plane

$\begin{array}{lllll}\text { Type } & \text { Serial } & \text { XP } & \text { YP } & \text { ZP } \\ \text { C } & 15 & -0.592 & 1.051 & 0.004 \\ \text { C } & 16 & -1.182 & -0.253 & -0.006 \\ \text { C } & 17 & -0.140 & -1.184 & 0.005 \\ \text { C } & 18 & 1.111 & -0.497 & -0.002 \\ \text { C } & 19 & 0.803 & 0.883 & -0.001\end{array}$

$\begin{array}{lllll}\text { Angle between plane } & 1 \text { and plane } & 2 \text { is } & 6.20 & \text { Degrees } \\ \text { Angle between plane } & 1 \text { and plane } & 3 \text { is } & 6.58 & \text { Degrees } \\ \text { Angle between plane } & 1 \text { and plane } & 4 \text { is } & 98.82 & \text { Degrees } \\ \text { Angle between plane } \quad 2 \text { and plane } & 3 \text { is } & 3.57 & \text { Degrees } \\ \text { Angle between plane } & 2 \text { and plane } & 4 \text { is } & 92.66 & \text { Degrees } \\ \text { Angle between plane } & 3 \text { and plane } & 4 \text { is } & 92.90 & \text { Degrees }\end{array}$

